\title{
Calibration of Equilibrium Tide Theory for Extrasolar Planet Systems
}

\author{
Brad M. S. Hansen ${ }^{1}$
}

\begin{abstract}
We provide an 'effective theory' of tidal dissipation in extrasolar planet systems by empirically calibrating a model for the equilibrium tide. The model is valid to high order in eccentricity and parameterised by two constants of bulk dissipation - one for dissipation in the planet and one for dissipation in the host star. We are able to consistently describe the distribution of extrasolar planetary systems in terms of period, eccentricity and mass (with a lower limit of a Saturn mass) with this simple model. Our model is consistent with the survival of short-period exoplanet systems, but not with the circularisation period of equal mass stellar binaries, suggesting that the latter systems experience a higher level of dissipation than exoplanet host stars. Our model is also not consistent with the explanation of inflated planetary radii as resulting from tidal dissipation. The paucity of short period planets around evolved A stars is explained as the result of enhanced tidal inspiral resulting from the increase in stellar radius with evolution.
\end{abstract}

Subject headings: planet-star interactions; planets and satellites: dynamical evolution and stability

\section{Introduction}

That tidal dissipation operates in extrasolar planet systems seems beyond dispute. The eccentricities of the closest planets are markedly smaller than those of planets with larger semi-major axes. However, the details of how tides sculpt the distribution, and the degree to which their influence extends in determining planetary radii is still a subject of active discussion. We have seen claims that the enhanced radii of some planets is due to recent or ongoing tidal dissipation, while others claim not (Jackson et al. 2008, 2009; Miller, Fortney \& Jackson 2009; Ibgui, Burrows \& Spiegel 2010; Leconte et al. 2010). We have also seen claims that some observed systems should be very short-lived (e.g. Hebb et al. 2010). Part of the problem is that the nature of tidal dissipation in these planets is still poorly understood. Furthermore, for some systems, dissipation of tides in the star is as important as that in the planet. Finally, much of the discussion has been couched in terms of the tidal $Q$ parameter, a traditional measure that has been calibrated in the solar system

\footnotetext{
${ }^{1}$ Department of Physics \& Astronomy and Institute of Geophysics \& Planetary Physics, University of California Los Angeles, Los Angeles, CA 90095, hansen@astro.ucla.edu
} 
(e.g. Goldreich \& Soter 1966) but which is frequency-dependent, and therefore sometimes difficult to translate from one system to another.

Our goal in this paper is to exploit the growing diversity of the exoplanet parameter space to try and place empirical constraints on the parameters of a specific tidal theory. We will adopt the equilibrium tide theory as our basic framework, although we recognise the distinct possibility that the physics of tidal interactions is much richer than this zeroth order treatment. We will also allow for the possibility that the differences in stellar and planetary structure require two different normalisations of the respective dissipative processes. Finally, we will cast our normalisations explicitly in terms of tidal dissipation constants $(\sigma)$, which are instrinsic measures of the star/planet internal structure and viscosity, and not dependent (within the context of this theory) on the frequency of forcing. Thus, we will be able to calculate a version of the tidal $Q$ that has a selfconsistent frequency dependence.

In $\S 2$ we present a brief summary of the tidal model we adopt, which we base on the model of Eggleton, Kiseleva \& Hut (1998). In $\S 3$ we review the literature on the related tidal dissipation problem of the circularisation period for solar mass main sequence binaries, and what this can tell us about the dissipation in solar mass stars. In $\S$ we will consider the calibration by fitting to the planetary distribution, in both period, eccentricity and planet mass. In $\S 5$ we will discuss the implications of our results, both in terms of observations and relative to other studies of this well-known subject.

\section{Tidal Model}

Eggleton, Kiseleva \& Hut (1998) - hereafter EKH - present a rederivation of the equilibrium tide model of Hut (1981), which is well suited to our purpose. It applies to arbitrarily large eccentricities (important given the observed distribution of planetary eccentricities around other stars), and and it isolates a physically motivated model of the dissipation in the planet or star, so that we may calibrate it for a specific class of object, instead of trying to cast our calculations in terms of the oft-used but physically obscure $\mathrm{Q} / \mathrm{Q}$ ' parameter 1 . We make the assumption that all of the host stars we discuss have the same basic internal dissipation constant $\sigma_{*}$, and that all the substellar bodies (planets and brown dwarfs) have the same $\sigma_{p}$. Once these quantities are specified, the mass and radii dependencies are explicit in the theory. Given this assumption, we restrict our attention to planets with masses greater than Saturn, to guarantee that the bodies are all of the same basic, fully-convective structure. Planets in the Neptune and SuperEarth categories may have sufficiently different internal structures that a different dissipation constant is required.

We also fold into our definition a dimensionless factor related to the structure of the object

\footnotetext{
${ }^{1} \mathrm{EKH}$ demonstrate that the formalism is equivalent to the 'constant time-lag' approximation often used in the derivation of tidal evolution equations.
} 
(since this scales with the same way as the dissipation constant). Thus, our definition of $\sigma_{*}$ and $\sigma_{p}$ includes the multiplicative factor $(Q /(1-Q))^{2}$, where $Q$ is the structure factor defined in EKH, and not the quality factor of Goldreich \& Soter. This is similar to the difference between the traditional definitions of $Q$ and $Q^{\prime}$, which are related by a factor determined by the Love number of the object in question, also determined by the density structure.

For aligned orbits, and dissipation in the planet, the semi-major axis a decreases at a rate determined by

$$
\frac{\dot{a}}{a}=-\frac{1}{T_{p}}\left[\frac{1+31 / 2 e^{2}+255 / 8 e^{4}+185 / 16 e^{6}+25 / 64 e^{8}}{\left(1-e^{2}\right)^{15 / 2}}-\frac{\Omega_{p}}{\omega} \frac{1+15 / 2 e^{2}+45 / 8 e^{4}+5 / 16 e^{6}}{\left(1-e^{2}\right)^{6}}\right],
$$

where $\mathrm{e}$ is the eccentricity of the orbit, $\omega$ is the angular frequency of the orbit, and $\Omega_{p}$ is the spin of the planet. The characteristic orbital decay time is

$$
T_{p}=\frac{1}{9} \frac{M_{p}}{M_{*} M} \frac{a^{8}}{R_{p}^{10}} \frac{1}{\sigma_{p}}
$$

where $M_{p}$ and $M_{*}$ are the planet and stellar masses, $M=M_{p}+M_{*}$ is the total mass, and $R_{p}$ is the planetary radius. The full equation for the orbital decay includes an equivalent contribution that results from dissipation in the star.

The eccentricity evolution is given by

$$
\frac{\dot{e}}{e}=-\frac{9}{2 T_{p}}\left[\frac{1+15 / 4 e^{2}+15 / 8 e^{4}+5 / 64 e^{6}}{\left(1-e^{2}\right)^{13 / 2}}-\frac{11}{18} \frac{\Omega_{p}}{\omega} \frac{1+3 / 2 e^{2}+1 / 8 e^{4}}{\left(1-e^{2}\right)^{5}}\right],
$$

and the rate of change of the spin is

$$
\frac{\dot{\Omega_{p}}}{\Omega_{p}}=\frac{\gamma}{2 T_{p}}\left[\frac{1+15 / 2 e^{2}+45 / 8 e^{4}+5 / 16 e^{6}}{\left(1-e^{2}\right)^{13 / 2}}-\frac{\Omega_{p}}{\omega} \frac{1+3 e^{2}+3 / 8 e^{4}}{\left(1-e^{2}\right)^{5}}\right],
$$

where $\gamma$ is the ratio of orbital angular momentum to spin angular momentum. As in the case of equation (1), each of these equations has an equivalent for dissipation in the star. The related timescale is

$$
T_{*}=\frac{1}{9} \frac{M_{*}}{M_{p} M} \frac{a^{8}}{R_{*}^{10}} \frac{1}{\sigma_{*}}
$$

An important feature of note in these equations is the strong dependence on stellar and planetary radius. As a result, we will need to properly account for the evolution of these quantities, as described below. In addition, we wish to choose appropriate normalisation constants for $\sigma_{p}$ and $\sigma_{*}$, based on the global properties of the objects involved. These dissipation constants scale as $\propto 1 /\left(\right.$ mass $\times$ length $^{2} \times$ time $)$. If we set the timescale to be the dynamical time of the object, we

\footnotetext{
${ }^{2}$ we retain this distinction because we will use the case $M_{p}=M_{*}$ in $\S 3$
} 
find that $\sigma_{0}=\left(G /\left(M R^{7}\right)\right)^{1 / 2}$. Using scalings relative to Jupiter and the Sun for planets and stars respectively, we define $\bar{\sigma}_{p}$ and $\bar{\sigma}_{*}$ using

$$
\begin{aligned}
& \sigma_{p}=5.9 \times 10^{-54} g^{-1} \mathrm{~cm}^{-2} s^{-1} \bar{\sigma}_{p} \\
& \sigma_{*}=6.4 \times 10^{-59} g^{-1} \mathrm{~cm}^{-2} s^{-1} \bar{\sigma}_{*}
\end{aligned}
$$

Finally, we note that the above formalism applies to aligned orbits, which is a special case of the general EKH formalism. We adopt this for the purposes of clarity, given that our principal interest is in the orbital distribution of the planets. There are indeed interesting questions related to the alignment of orbits and stellar spin rates, but are more particularly applicable to questions of the origin of planetary eccentricities. For the purposes of this calculation, we adopt an eccentricity distribution as an initial condition and leave questions of origin to a later date.

\subsection{Stellar and Planetary Evolution}

The strength of the tidal force between fluid bodies is a strong function of the radii, and thus we need to treat the evolutionary history of stellar and planetary radii correctly. In particular, we will find in $\S 3$ that the radius evolution for the star is a non-negligible contributor, including the pre-main sequence stage. Therefore, in the calculations to follow, we use the evolutionary models of Baraffe et al. (1998) to describe the evolution of stellar radius with age, as a function of mass. The evolution for some representative stars is shown in Figure 1.

We also need to describe the evolution of the planetary radius with mass. We use the models and formalism described in Hansen \& Barman (2007). The thermal evolution of the planet is calculated using a Henyey code, with boundary conditions based on models for irradiated atmospheres. The result is that the rate at which the radius shrinks is slowed as the planet experiences more irradiation, as has been described by a variety of authors (Guillot et al. 1996; Burrows et al. 2000; Baraffe et al.2003). We also include a bulk heating contribution, to account for energy input due to tidal dissipation in the planet. This is included as a bulk heating rate per unit mass. Figure 2 demonstrates the various effects. The solid curve shows a planet (with no core) with appropriate mass $\left(1.41 M_{J}\right)$ and irradiation to serve as an analogue to WASP-12. We choose this object because it has been modelled by both Miller et al. (2009) and Igbui et al (2009), and can thus serve as a useful baseline for comparison with the models in those papers. The figure shows the radius (with appropriate correction for the transit radius measurement). Inspection of the two other papers shows that our results lie in between those of Miller \& Ibgui (after correcting for the fact that the Miller model includes a $10 M_{\oplus}$ core). The dotted and dashed lines show the radius evolution of the same planet but including the effects of tidal heating. In calculating these curves we have parameterised our model as best we could (given the different formalisms) to match the parameters used in Miller et al. (dotted) and Ibgui et al (dashed). The parameters were chosen to yield the same $Q^{\prime}$ values at the current separation of WASP-12. We see that we reproduce the character of 
the Miller result, because we incorporated a fixed floor of eccentricity ( $e=0.05)$, which maintains the tidal dissipation in the planet and causes runaway heating at small separations. The dashed line lies above the solid line, indicating that tidal heating is playing a role, but we do not reproduce the increase in radius at late times in the Ibgui model, because our model circularises the orbit much earlier, even with the adjusted parameterisation. We discuss the reasons for this further in $\S 5$.

The above comparison indicates that the planetary models used in the various studies are similar, if not exact, and that most of the differences in the final comparison of radii are likely to result from the treatment of the tidal evolution, which is the motivation for this paper.

\section{Normalisation of Stellar Binaries}

We would like to calibrate the stellar dissipation constant independently of the planets by examining the circularisation of stellar mass binaries. We follow the formalism of Meibom \& Mathieu (2005) - hereafter MM05 - to calibrate the model. We start with a gaussian initial distribution of eccentricities, with mean value $\bar{e}=0.35$ and dispersion $\sigma_{e}=0.21$. This represents an estimate of the eccentricity distribution of long-period (>50 days) binaries in the Pleiades, M35, Hyades/Praesepe, M67 \& NGC 188. Initial periods are drawn from a log-normal distribution and the evolution is calculated using the formalism in $\S 2$. Spin periods for the stars are initially assumed to be small (30 days), and the evolution of the stellar radius is calculated as in $\S 2.1$. MM05 found that the most robust estimate of the tidal circularisation period $P^{\prime}$ was to fit a function of the form

$$
e(P)=0.35\left(1-e^{0.14\left(P^{\prime}-P\right)}\right)
$$

to the final distribution (where $\mathrm{e}=0$ for $P<P^{\prime}$ ). MM05 provide measures of the circularisation period for a series of open clusters and representative field populations, reproduced in Table 1 ,

Using the above procedure, we simulate the tidal evolution of a population of $1 M_{\odot}$ stars with a variety of values of $\bar{\sigma}_{*}$ and fit the same function as MM05 to the result. By comparing to the observationally determined circularisation period, we can then infer the empirical values of $\sigma_{*}$ which correspond to the observations within the context of the equilibrium tide model. The inferred values are included in Table 1. Figure 3 shows the evolution of the circularisation period with age, along with that expected from our model, adopting the best fit value of $\bar{\sigma}_{*}=5.3 \times 10^{-5}$. We see that the equilibrium tide model provides an excellent description of the overall trend of the circularisation period with astrophysical age. We also see that the pre-main sequence stage is essential, as asserted by Zahn \& Bouchet. The dotted line shows the evolution of the circularisation period if radius is held fixed, and calibrated to fit the data at late times. At earlier times this calibration dramatically underestimates the amount of circularisation. Essentially, the circularisation period is set by the pre-main sequence dissipation for populations with ages $<2 \mathrm{Gyr}$ and only begins to increase, due to the main sequence dissipation, at larger ages. The best fit $\bar{\sigma}_{*}$ is also an order of magnitude smaller than would have been inferred without the pre-main sequence evolution. 


\section{Exoplanets}

As has been discussed by several authors, the circularisation of planetary orbits can be affected by dissipation in both the planet and the star. In our model, we allow for a different dissipation constant in the two. We use the above calibration for $\bar{\sigma}_{*}$ and now wish to examine whether we can explain the observed exoplanets with a single estimate for $\bar{\sigma}_{p}$.

One difference in philosophy between analysing the stellar binaries and the exoplanet systems is that, in the latter, there is good reason to believe that some systems may have had circularity imposed by formation in a disk. As such, modelling the full eccentricity distribution is problematic without a convincing model for the underlying original functional form. Therefore, we proceed with a two pronged approach. First we will analyse the exoplanet distribution in the same manner as the stellar binaries in $\S 3$. This yields an initial ballpark estimate. To refine this, we will then repeat the exercise by modelling the individual systems that define the upper envelope of the period-eccentricity relation. The exoplanet distribution is now sufficiently well sampled that this is a reasonably well-defined locus, and the accumulated data from fitting the individual systems should indicate the appropriate value of $\bar{\sigma}_{p}$.

\subsection{Jupiters}

To begin our treatment of the exoplanet sample, we want to define a sample of exoplanets in the same spirit as those of $\S 3$. There are several pitfalls in such an analysis. The exoplanet sample covers a range of ages and stellar populations, and is compiled through a variety of search techniques with different biases. Nevertheless, a global analysis will at least indicate a initial guess for the dissipation rate, that can set later refinements in context.

We restrict our initial calculation to planets with a factor of three of Jupiter's mass, i.e. 0.3$3 M_{J}$, orbiting stars of $1 M_{\odot}$. The lower bound serves to restrict our analysis to objects that share the same basic internal structure of a fully convective, $\mathrm{H} / \mathrm{He}$ mixture. The upper bound is somewhat arbitrary, but the observations of eccentricity versus mass suggest that this lies close to a qualitative difference in behaviour. Figure 4 shows the period-eccentricity relation for this

sample, in a comparison with the M35 solar mass sample of MM05. Clearly we expect a shorter circularisation period in the case of exoplanets.

We use the same initial distributions as in $\S$ 3, except for the initial eccentricity distribution, and describe the evolution of stellar and planetary radii using the formalism of $\S$ 2.1. Following in the spirit of the stellar sample, we compile the distribution function of eccentricities for planets in the above mass range by using those systems with orbital periods from 50 to 1000 days. This is fit by a gaussian, with a somewhat lower mean value (0.2) than for the stellar sample, and with a slightly higher dispersion (0.25). We fit the resulting tidally evolved population with a function

$$
e(P)=0.2\left(1-e^{0.65\left(P^{\prime}-P\right)}\right)
$$


that is similar in spirit to the one of MM05, but with a sharper rise as dictated by the population that evolves from the model. Fitting a similar function to the data yields a circularisation period of 2.85 days. We refer the reader again to the discussion in MM05 regarding the choice of this function, which is designed to trace the mean trend of eccentricity with period, not the largest circular orbit.

We add one additional step to the comparison over the procedure followed in MM05. We restrict our fitting function to systems with $e>0.02$. This was necessary because the planet sample has a significant eccess of circular orbits above the circularisation period over that expected from a model population drawn from a tidally evolved model population. In principle, this could indicate a real eccess, suggesting that some planets do migrate inwards on circular orbits, but could also be significantly affected by the selection effects associated with radial velocity and transit surveys. Certainly there is no circular excess in the range of orbital periods $>10$ days. Thus, we restrict our model comparison to the distribution of non-circular orbits.

Performing the model comparison, for a mean sample age of $3.0 \mathrm{Gyr}$, we obtain $\bar{\sigma}_{p}=6.8 \times$ $10^{-7}$. Figure 5 shows the comparison between the observed distribution and the model population. This provides us with an approximate mean estimate, but better constraints may be obtained by comparing to individual objects with well-determined parameters. To this end, we can consider several specific objects whose properties define the approximate envelope of the P-e relation.

\subsection{Individual Objects}

To refine the constraints on $\bar{\sigma}_{p}$, we wish to examine individual systems. By modelling those that define the envelope of the period-eccentricity relation, and by addressing the particular system parameters (stellar mass, age, etc), we hope to refine our initial estimate. In contrast to the calculations of $\S 4.1$, we perform the following calculations for the values of stellar and planetary mass specific to each system. We furthermore assume the stars have a stellar rotation of 30 days period initially, and discuss the effects of and on stellar spin in $\S 5.4$.

\subsubsection{WASP-17b}

The shortest period planet with claimed eccentricity $>0.1$ is WASP-17b (Anderson et al. 2010). This planet has a mass of half that of Jupiter and an age estimated to be $3_{-2.6}^{+0.9}$ Gyr. The eccentricity is $0.13_{-0.07}^{+0.11}$. The allowed values of $\bar{\sigma}_{p}$ depend somewhat on the assumed age. If the age lies at the low end of the stated range, there is not much time to circularise all possible orbits, and dissipation rates as high as $\bar{\sigma}_{p}=1.2 \times 10^{-6}$ allow an eccentricity of this magnitude to survive. At the upper end of the age range, this dissipation rate is unacceptably large and would circularise the orbit completely. In this case an upper limit of $\bar{\sigma}_{p}=6.8 \times 10^{-8}$ is determined. Furthermore, with an abundance of planets in the period range, it is likely that eccentricities much larger than 
0.24 are also disallowed. This can be used to place a lower limit on the dissipation rate as well, leading to an overall constraint (including age uncertainty),

$$
6.8 \times 10^{-8}<\bar{\sigma}_{p}<1.2 \times 10^{-6} .
$$

Thus, WASP-17b alone allows an order of magnitude variation in the estimated dissipation, driven mostly by the uncertainty in the age.

\subsection{2. $B D-103166$}

This planet has a mass similar to WASP-17b and a slightly smaller semi-major axis. The measured eccentricity (Butler et al. 2000) is $e=0.07 \pm 0.05$, so we consider the possibility that the eccentricity is as large as 0.12 . The age determined from chromospheric activity (Saffe, Gomez \& Chavero 2005) ranges from 0.5-4.2 Gyr, depending on the calibration used. Furthermore, the claimed radius of $1.7 R_{\odot}$ is rather large for a $1 M_{\odot}$ star, suggesting that the age could be closer to $\sim 10 \mathrm{Gyr}$.

If the system is $\sim 10 \mathrm{Gyr}$ old, then we can use the $1 \sigma$ lower limit on the eccentricity to constrain $\bar{\sigma}_{p}<1.7 \times 10^{-6}$. Dissipation stronger than this would have circularised the orbit over this time span. Similarly, a lower limit can be obtained by requiring that the $1 \sigma$ upper bound on the eccentricity match the simulations at the shortest estimated age $(0.5 \mathrm{Gyr})$. Once again, this leaves us with an order of magnitude uncertainty, dictated by the uncertain age of the star.

$$
3.4 \times 10^{-7}<\bar{\sigma}_{p}<1.7 \times 10^{-6} .
$$

Another possible constraint in the same period range is possible from the system WASP-6. In this case the error bars on the eccentricity are smaller, but the age uncertainty is even larger, and the dissipation constraints are not markedly better.

\subsubsection{Kepler- $6 b$}

Clearly, one of the big uncertainties in this estimate is the time that tides have had to exert their influence in these systems. Planets discovered by Kepler will be particularly interesting for this purpose, because their asteroseismological analyses can potentially constrain the ages much better. Kepler has yet to announce a planet with a measureably eccentric orbit, but we can get an estimate of what constraints might be possible by considering the system Kepler-6b. In the fits to the radial velocity data for Kepler-6b, the orbit was assumed to be circular (Dunham et al. 2010), and the data appear consistent with this assumption. If we adopt an illustrative constraint of $e<0.05$ and the calculated age of $3.8 \pm 1 \mathrm{Gyr}$ for this $0.67 M_{J}$ planet, we obtain a lower limit

$$
\bar{\sigma}_{p}>3.4 \times 10^{-7}
$$


for the tidal dissipation. This is the amount required to circularise the planet to $e<0.05$ within 5 Gyr. As Kepler accumulates more data, a better calibration is likely.

\subsubsection{COROT-5}

The system COROT-5b offers an improvement, by virtue of a reasonably constrained spectroscopic age (Rauer et al. 2009) of $6.9 \pm 1.4$ Gyr. The planet is once again roughly half a Jupiter

mass around a solar mass star. The eccentricity is $e=0.09_{-0.04}^{+0.09}$, at an orbital period of 4.03 days. The range of $\bar{\sigma}_{p}$ compatible with this system's error bars and age range is

$$
1.2 \times 10^{-7}<\bar{\sigma}_{p}<6.8 \times 10^{-7}
$$

\subsubsection{HD118203b}

Tracing the upper envelope of the eccentricity-period relation, we find the system HD 118203b, which has a mass of $2.13 M_{J}$, a period of 6.1 days and an eccentricity $0.31 \pm 0.01$ (Da Silva et al. 2005). The age of the system is estimated to be $4.6 \pm 0.8 \mathrm{Gyr}$ (Da Silva et al. 2010). In order for such a planet to possess this level of eccentricity at these ages, it places an upper limit on the level of dissipation of

$$
\bar{\sigma}_{p}<3.4 \times 10^{-6} .
$$

This is obtained by requiring that the envelope of the $\mathrm{P}$-e relation intersect the observed value at an age of 3.8 Gyr. In principle, if this object traces the upper edge of the eccentricity distribution, we could also place a lower limit. However, the envelope does not move significantly up even if $\bar{\sigma}_{p}$ is reduced to zero, because stellar tides become more important as the planet mass increases. We will discuss this further below.

\subsubsection{HD185269}

This is a planet orbiting a subgiant (Johnson et al. 2006), with an eccentricity of $0.3 \pm 0.04$. Johnson et al. quote an age (4.2 Gyr) with no error bar, so we estimate an error bar of \pm 0.3 Gyr on the basis of the error in the estimated mass and radius, and our stellar models. The subgiant nature of this star leads to some interesting tidal behaviour. For the maximum estimated age (4.2 Gyr), we find that we cannot match the observed parameters, even if $\bar{\sigma}_{p}=0$. This is because dissipation in the star is strong enough to circularise orbits at this separation. However, at the lower end of the age range $(3.9 \mathrm{Gyr})$, the stellar tides are sufficiently weakened that values as large as $\bar{\sigma}_{p}=5.1 \times 10^{-6}$ are consistent with the data. This dramatic change is because the radius of the star is increasing as it evolves towards the giant branch, making the stellar tides increase in 
strength. The end result is that the constraint is much the same as before

$$
\bar{\sigma}_{p}<5.1 \times 10^{-6} .
$$

\subsubsection{Synthesis}

Fitting each of these systems yields a range of values consistent with the observed parameters. It is possible to achieve a successful synthesis of these various constraints with a value

$$
\bar{\sigma}_{p}=5 \pm 2 \times 10^{-7}
$$

and

$$
\sigma_{*}=1.3 \times 10^{-5}
$$

It is encouraging that $\bar{\sigma}_{p}$ derived in this manner is similar to that from the fit of the 'average' model to the global distribution. The consistency suggests that neither approach is overly bedeviled by systematic issues related to, for example, the age of the system in question.

\subsection{Massive Planets}

In $\S 4.1$ we restricted our attention to planets less massive than $3 M_{J}$, and calibrated the planetary dissipation from the observed distribution. There is potentially also interesting information in the more massive planets, as shown in Figure 6. In the lower panel, we show the period-eccentricity distribution of the Jupiter mass sample in Figure 5. In the upper panel we show the same relation for planets with $M>3 M_{J}$. The upper envelope of the distribution appears shifted to lower periods in the latter sample, suggesting a useful test of our equilibrium tide model. Can we explain this shift with our calibration?

\subsubsection{WASP-14b}

The planet WASP-14b (Joshi et al. 2008) would appear to be a particularly useful system, as it has a measured eccentricity of $0.090 \pm 0.003$ (recently confirmed by Husnoo et al. 2010) at an orbital period of 2.24 days - at which lower mass planets are definitely circularised. The planet mass is $7.73 M_{J}$ and the estimated age of the system is $0.75 \pm 0.25$ Gyr.

When we adopt our calibrated model, it fails spectacularly to match the observed values of WASP-14b. The observed parameters of WASP-14b lie well above and to the left of the allowed period-eccentricity region, for systems with the mass and age observed. Reducing the amount of dissipation in the planet (even to zero) does not change the envelope significantly, so it is not our assumption that dissipation is the same across all planet masses that is the problem. Rather, it 
is the dissipation in the star that has to be reduced. The larger mass planet raises stronger tides on the star and this causes the stellar tides to drag the planet in further. A planet with the mass, period and eccentricity of WASP-14b should have been swallowed by the star on a timescale shorter than the estimated age of the system.

\subsection{2. $X O-36$}

To further illustrate the problem, let us consider the system XO-3b (Johns-Krull et al. 2008; Winn et al. 2008). This planet has a mass of $11.8 M_{J}$, which is almost large enough to be a brown dwarf. With an orbital period of only 3.2 days, it nevertheless has an eccentricity of $0.26 \pm 0.02$ and

an estimated stellar age of $2.8_{-0.8}^{+0.6} \mathrm{Gyr}$. With these parameters and the nominal calibration from lower masses, XO-3b would have to have an orbital period of 6.6 days to survive for the estimated age. As in the case of WASP-14b, changing the planetary dissipation rate does little to alleviate the mismatch, and we are again faced with the requirement to reduce the dissipation in the star.

\subsection{Recalibration of the stellar dissipation}

Testing our calibration of $\S 4.1$ on the more massive planets shows that we cannot get a consistent calibration of the equilibrium tide model that applies to both stellar binaries and planetary systems. The reason is that the level of stellar dissipation required to match the circularisation in stellar binaries is too strong to match the planetary systems. We will return to the implications of this is $\S$, but, for now, let us consider a recalibration of the stellar tides using just the planetary systems.

\subsubsection{HAT-P-2b}

At longer orbital periods (5.6 days), we find the planet HAT-P-2b (Bakos et al. 2007), with mass $9.1 M_{J}$ and eccentricity $0.517 \pm 0.033$, at a system age of $2.7 \pm 0.5 \mathrm{Gyr}$. This is perhaps the most extreme of the known systems, and can thus serve as a useful system to recalibrate the stellar dissipation. We simulate this system using the individual system parameters, as in previous cases, with one exception. This system is unique amongst those considered up to this point in that it has a stellar rotation that is faster than the planetary orbital rotation, so that the stellar tidal effects could potentially push the planet outwards rather than inwards. For consistency with our previous models, we will assume a stellar rotation period of 30 days initially in our calculations. We return to this system in $\S 5.4$ and demonstrate that the spin turns out to not be quantitatively important.

If we set dissipation in the planet to zero, the maximum acceptable stellar dissipation is $\bar{\sigma}_{*} \sim 1.6 \times 10^{-7}$. If we use our nominal planetary dissipation of $\bar{\sigma}_{p} \sim 5 \times 10^{-7}$, we need to reduce $\bar{\sigma}_{*}$ 
by only a further factor of 2 . This indicates that the massive planet systems are mostly sensitive to $\bar{\sigma}_{*}$ and only weakly sensitive to $\bar{\sigma}_{p}$. Thus, we will adopt a provisional calibration of

$$
\bar{\sigma}_{p}=5 \times 10^{-7}
$$

and

$$
\sigma_{*}=8 \times 10^{-8}
$$

If we apply these numbers to the systems XO-3b and WASP-14b we get satisfactory fits as well, shown in Figure 7. The reduction in the strength of the stellar tide means that planets can now orbit closer to the star without being pulled inward and swallowed.

Figure 8 demonstrates how the relative strength of stellar and planetary tides change with the planet mass. The two curves show two evolutionary scenarios. The initial condition was chosen such that it would yield the correct period, eccentricity and age for the XO-3b system, using our final parameterisation. The lower curve is calculated using all the same parameters and starting conditions, but reducing the mass of the planet to $1 M_{J}$. We see that the lower mass planet is more rapidly circularised, and at larger distances. The consequence is that the periastron for the lower mass planet actually moves outwards as the orbit circularises, reducing the strength of the stellar tide. On the other hand, the periastron for the real XO-3b moves inwards, because the stellar tide is dragging the planet inwards at a rate comparable to the rate at which the planetary tide is circularising the orbit.

\subsubsection{Revisiting the Jovian mass systems}

Comparing the new calibration to the same jovian-mass planets as before shows that most are consistent with the new calibration. One system that is not is COROT-5b, which requires a slight reduction in $\bar{\sigma}_{p}$ for consistency with the new value of $\bar{\sigma}_{*}$, yielding a final calibration

$$
\bar{\sigma}_{p}=3.4 \times 10^{-7}
$$

and

$$
\bar{\sigma}_{*}=7.8 \times 10^{-8}
$$

\subsection{Closest Planets}

Our re-calibration of the stellar dissipation results in a reduction of the strength by a factor of 160 , relative to what is necessary to explain the circularisation of binary stars. We can also attempt to constrain the strength of tidal dissipation by examining the survivability of very close planets. Planetary tides will act to circularise an orbit, but reduce to zero once this has occurred. Dissipation in the star, on the other hand, acts to transfer angular momentum from the planetary 
orbit to the stellar spin, and will slowly drag a planet inwards, even after it has circularised. Thus, we can constrain $\bar{\sigma}_{*}$ independently by examining the expected lifetime of known planets.

In the limit of a circular orbit, we can calculate a characteristic inspiral time

$$
T_{i n}=8.2 \times 10^{9} \text { yrs }\left(\frac{a}{0.02 A U}\right)^{8}\left(\frac{R}{R_{*}}\right)^{-10}\left(\frac{M_{p}}{M_{J}}\right)^{-1}\left(\frac{\bar{\sigma}_{*}}{7.8 \times 10^{-8}}\right)^{-1} .
$$

Table 2 shows the characteristic inspiral times for all the planets interior to $0.03 \mathrm{AU}$ (excluding those that orbit $\mathrm{M}$ dwarfs, for whom the stellar tide might be markedly different). We see that the shortest ages are for WASP-18b and WASP-12b, but that both are still $>10^{8}$ years and consistent with the ages estimated for the systems as a whole. The only formal conflict is for OGLE-TR-56, whose stated age is $>2 \mathrm{Gyr}$, but whose estimated inspiral time is $1.1 \mathrm{Gyr}$, and so is broadly consistent given the systematics of stellar age determination for such stars. The fact that the observed systems can survive for a reasonable time using this parameterisation is encouraging. We note also that reducing the ages in Table 2 by a factor of 160 would result in significant age conflicts for many of the the systems listed there - another indication that the calibration using the stellar binaries is too large.

It is also of interest to consider the distribution of planets with semi-major axis. Figure 9 shows the cumulative distribution of jovian mass planets (detected from transits only in this case), compared to the same distribution from our simulations. The original semi-major axis distribution was distributed logarithmically, and the original eccentricity distribution was chosen to be the same as in $\S 4.1$. We show four models. The leftmost is the distribution left if we assume there is no dissipation in the star. The dotted histogram indicates the distribution after 0.1 Gyr, using the dissipation in equation (4.4.2). The short dashed histogram shows the same population but after 4 Gyr. Finally, the rightmost histogram shows the distribution after 0.1 Gyr in the case where the stellar dissipation is given by the original value in $\S 3$. In all four cases, the theoretical planets were weighted by $1 / \mathrm{a}$, in a crude attempt to account for their detectability in transit surveys. This is an admittedly very crude model, subject to question in terms of both the initial semi-major axis and eccentricity distributions, but demonstrates how one might go about constraining $\bar{\sigma}_{*}$ once a better understanding of the observational selection effects is available. It also demonstrates that the data are more consistent with a simple model incorporating a small amount of stellar dissipation rather than no dissipation at all or that found by our stellar binary calibration.

\subsection{Neptunes}

In principle, our calibration should extend down to lower masses, if they are accurately described as cosmic abundance $\mathrm{H} / \mathrm{He}$ mixtures. However, the current transitting planet sample does not contain any planet with mass $<0.2 M_{J}$ that has a radius $>0.5 R_{J}$. Thus, the currently known

Neptune-mass objects likely all have significant core fractions, which may dramatically influence the dissipation and may change $\bar{\sigma}_{p}$. 


\subsection{Age Dependance}

With a calibration of the tidal dissipation in hand, one can also examine how the distribution of planets evolves with time. Figure 10 shows the period distribution of a model population of $1 M_{J}$ planets around a $1 M_{\odot}$ star, comparing the orbit period at an age of 0.1 Gyr with that at 1 Gyr. The relation deviates from linear at periods $<1.5$ days, showing that it is planets with orbital period in this range that are expected to evolve over the evolutionary course charted by extant observations. The spread at periods $>2.5$ days is related to the amount of circularisation that takes place over the same timespan. Another way of phrasing this relation is that planets observed in a 1 day orbit around a 0.1 Gyr old star will spiral into the star within 1 Gyr.

\section{Discussion}

The issue of planetary tidal evolution has a long and illustrious history, with many applications to our own solar system (e.g. Peale 1999). The importance of tides was realised immediately upon the discovery of the first extrasolar planets (e.g. Rasio et al. 1996; Marcy et al. 1997) because of the extremely short periods of the first planets discovered using radial velocities. The interest in tidal effects has been unwavering ever since, especially since the advent of transit surveys, which strongly favour the detection of short period planets. As such, our results have relevance to many other studies (and vice versa). However, before we can compare and contrast our results with those of others, we need to provide a translation between the traditional manner of parameterising the dissipation using ' $Q$ ', and our intentionally different approach.

\subsection{The $Q^{\prime}$ Parameter}

As a large fraction of the literature on this subject is phrased in terms of a particular value of the tidal dissipation parameter $Q^{\prime}$, it is of interest to see how our model compares. If we compare of our expression with an equivalent expression cast in terms of $Q^{\prime}$, such as Jackson et al (2008), we obtain an expression

$$
Q_{p}^{\prime}=\left(\frac{G}{M_{*}}\right)^{1 / 2} \frac{a^{3 / 2}}{R_{p}^{5} \bar{\sigma}_{p}}=\frac{G}{\omega} \frac{1}{R_{p}^{5} \sigma_{p}}
$$

where $\omega$ is the orbital angular frequency. Putting our best-fit numerical values into this yields

$$
Q_{p}^{\prime}=3.0 \times 10^{8}\left(\frac{a}{0.1 A U}\right)^{3 / 2}\left(\frac{R_{p}}{1 R_{J}}\right)^{-5}\left(\frac{M_{*}}{M_{\odot}}\right)^{-1 / 2} .
$$

Note that this is no longer a constant, but has a dependence on both semi-major axis and planetary radius (although not planetary mass). The radius dependence is quite strong and this is, in part,

the reason for the strong effect of tidal dissipation. In general, $Q^{\prime}$ will drop as the planet approaches the star and dissipation will increase. 
An equivalent analysis for the dissipation in the star yields an expression for the $Q$ of the star,

$$
Q_{*}^{\prime}=6.3 \times 10^{8}\left(\frac{a}{0.1 A U}\right)^{3 / 2}\left(\frac{R_{*}}{1 R_{\odot}}\right)^{-5}\left(\frac{M_{*}}{M_{\odot}}\right)^{-1 / 2} .
$$

Figure 11 shows the resulting values of $Q_{p}^{\prime}$ and $Q_{*}^{\prime}$ for the close planet sample. We see that a large fraction of the close Jupiter systems are characterised by present day values $Q_{p}^{\prime} \sim 3 \times 10^{7}$, although the closest systems (a few are labelled in the plot) show values a little smaller. The range of values of $Q_{*}^{\prime}$ shows somewhat greater variation, presumably because the strong dependence on $R_{*}$ means that $Q_{*}^{\prime}$ can vary by an order of magnitude with a $60 \%$ change in radius. The functional form of equations (11) and (12) can be easily understood by analogy with the simple harmonic oscillator description of the equilibrium tide model (Greenberg 2009). Many authors have estimated $Q^{\prime}$ values for individual systems based on various observational constraints and model requirements (e.g. Matsumura, Takeda \& Rasio 2008; Miller et al. 2009; Ibgui et al. 2010). These are often significantly different than the corresponding values above, suggesting that efforts like this one to enforce consistency within the basis of a single physical model will be increasingly necessary as the observed sample grows.

One of the traditional cornerstones of estimating tidal dissipation in giant planets is to draw a comparison with the constraints on the dissipation in Jupiter, inferred by assuming that the resonant configuration of the Gallilean satellites is driven by tidal dissipation in Jupiter (Goldreich \& Soter 1966). To cast our results in an appropriate form for this comparison, we must re-arrange terms in the formalism of $\S 2$, moving Jupiter to the role of 'Star', and Io to the role of planet. Furthermore, the forcing frequency $\left(\Omega_{J}\right)$ of this system comes from the rotation of Jupiter, not the orbit of Io, so that we need adopt the limit of $\Omega_{J}>\omega$. Once again, in this limit, we infer

$$
Q_{J}^{\prime} \sim \frac{1}{2} \frac{G}{\Omega_{J}} \frac{1}{R_{J}^{5} \sigma_{p}} \sim 5 \times 10^{6}
$$

for a rotation period of 10 hours and $\sigma_{p}=2 \times 10^{-60} \mathrm{~g}^{-1} \mathrm{~cm}^{-2} \mathrm{~s}^{-1}$. This is only slightly higher than the usually quoted range of $Q_{J} \sim 10^{5}-10^{6}$. Of course, it is also possible that the Laplace resonance of the satellites is primordial (Peale \& Lee 2002), in which case there are no constraints on $Q_{J}^{\prime}$ from the Io system. This value is somewhat larger than the $Q^{\prime} \sim 4 \times 10^{4}$ inferred by Lainey et al. (2009) from a long-term astrometric monitoring program, suggesting that a stronger frequency dependance may be required at higher forcing frequencies 3 .

\footnotetext{
${ }^{3}$ It is interesting that the factor of 50 difference between our $Q_{J}^{\prime}$ and the observation of Lainey could be explained if $\sigma_{p}$ scales quadratically, as in the Goldreich-Nicholson formalism - assuming the conversion is from 3 days to 10 hours.
} 


\subsection{Tidal Inflation}

One of the principal motivations for our study was the recent flurry of interest in whether tidal dissipation can contribute to the inflated radii observed for some planets. Several authors have attempted to account for the enhanced radii of certain planets by invoking the dissipation due to tides to heat the planet internally. Jackson et al. (2008a,b) have run the evolution of various systems backwards and claim that, for reasonable choices of $Q$, one could explain the tidally inflated radii of some planets as the result of residual heat left over from recent circularisation. However, they do not treat the evolution of the planetary radius self-consistently, an important omission, as the strength of tides can be strongly influenced by the radius. To do this correctly required forward modelling, and calculations of this type have been recently performed (Miller et al. 2009; Igbui et al. 2010). Miller et al. find that tidal inflation may explain some systems, but not all. Igbui, Spiegel \& Burrows (2009) find consistent solutions for systems such as WASP-4b and WASP-12b with values of $Q_{p}^{\prime} \sim 10^{8}$ and $Q_{*}^{\prime} \sim 3 \times 10^{6}\left(P_{\text {orb }} / 10 \text { days }\right)^{-1}$, (which amounts to $Q_{*}^{\prime} \sim 2.5 \times 10^{7}$ for these two systems). One concern about such studies is that it is not clear how the choices of $Q$ should be related between different systems. One of the motivations for this current study was to see what a consistent formalism might yield. Another concern is that the tidal evolution equations in the above papers were truncated at second order in the eccentricity, even when the eccentricity was large. This approach has been (rightly) criticised recently by Leconte et al. (2010), who use a set of equations derived from the model of Hut (1981). This study is the closest extant one to ours, both in spirit and execution (since the EKH model is also ultimately derived from the same model as that of Hut).

With our final calibration, tidal inflation is not a likely cause of planetary inflation. Figure 15 shows the evolution for two prominent inflated planet systems, WASP-12 and WASP-4. In each case, the initial parameters were chosen such that the forward evolution with the parameterised model would yield the correct semi-major axis and eccentricity at the estimated age of the system. In both cases (and all others we investigated) the damping of the eccentricity does cause inflation of the planet, but happens on short times $\left(\sim 10^{7}\right.$ years $)$, so that the planetary cooling reduces the radius to more traditional values by the time the planet reaches it's current semi-major axis. Discrepancies are even larger for more distant inflated systems (like Tres-4), which experience little tidal evolution in this model. The only way tidal inflation could explain these radii is if the planetary orbit was circularised only within the last $\sim 10^{8}$ years, requiring a late injection of the planet orbit. In this we are in complete agreement with Leconte et al, who find a similar disagreement with previous studies, and ascribe it to the fact that the truncation of the tidal equations to quadratic order weakens the strength of the tide, allowing the planet to dissipate energy at later times, and increasing the amount of tidal inflation. This is ultimately why we cannot reproduce the evolutionary history of Igbui et al. (2010) in Figure 2, no matter what parameterisation we adopt.

Given the similar philosophy, it is of interest to compare the values of $Q^{\prime}$ assumed in Leconte et al. with our calibrations. The Leconte model assumes a constant time lag, which EKH showed is an equivalent assumption to that of a bulk dissipation constant such as we use here. As a result, 
their $Q^{\prime}$ values have the same frequency dependence as ours, and they have normalised them by assuming different values at a period of 1 day. We can use equation (12) to normalise our model in a similar fashion. For our calibration, $Q_{p}^{\prime} \sim 10^{7}$ and $Q_{*}^{\prime} \sim 6 \times 10^{7}$ at 1 day periods (with some scatter resulting from the radius dependence in our model). Thus, our planetary dissipation is comparable to the upper range assumed by Leconte et al, although our stellar dissipation is an order of magnitude weaker than the range they studied.

For completeness, we should note that our inability to find a reasonable tidal inflation model is confined to the simple case of single planet tidal evolution. Some studies (e.g. Bodenheimer et al. 2001; Mardling 2007; Batygin, Bodenheimer \& Laughlin 2009; Ibgui et al. 2010) invoke a third body to perturb the eccentricity and maintain some level of tidal inflation. Figure 16] shows the evolution of the WASP-12b system in our tidal model with the addition of an eccentricity floor for the planet. As the stellar tide starts to drag in the orbit, the continued dissipation in the planet does indeed inflate the radius, and could potentially explain WASP-12b if $e>0.02$. This is consistent with the initial claims for the system (Hebb et al. 2009; Lopez-Morales et al. 2009), but is not consistent with more recent measurements (Campo et al. 2010; Husnoo et al. 2010).

In conclusion, we find that it is difficult to explain the inflated planetary radii within the context of an equilibrium tide model parameterised to match the overall properties of the exoplanet distribution, especially if one uses a tidal evolution model that treats large eccentricity systems selfconsistently.

\subsection{The Lack of Hot Jupiters around subgiant A stars}

Searches for planets around more massive stars $\left(>1.5 M_{\odot}\right)$ report a higher frequency of planets, but a conspicuous lack of close-in planets (Johnson et al. 2007; Lovis \& Mayor 2007; Sato et al. 2008; Niedzielski et al. 2009). However, the recent report of a likely transitting planet in a 1.2 day

orbit around the $1.5 M_{\odot}$ star HD15082 (Collier Cameron et al. 2010) suggests that the existence of such planets is possible at least during the main sequence stage. The fact that the radial velocity searches are performed around subgiants (so that the atmospheres are slowly rotating and more amenable to radial velocity measurement) means that one can speculate whether the close-in planets have been swallowed as the result of the evolution of the central star. Johnson et al. (2007) discount this possibility because the stellar radii do not approach the semi-major axes of the hot Jupiters until much later stages.

However, we have seen that the stellar tidal coupling can cause a planet to spiral inwards. Can this process, with our stellar dissipation calibration, explain the paucity of hot Jupiters around Atype subgiants (e.g. Sato et al. 2008)? Figure 12 suggests that tidal effects will contribute, at least partially, to the removal of close-in planets. We can determine the degree to which tides can drag in planets, as a function of stellar radius, by integrating the tidal evolution in concert with a model for the stellar evolution, and noting at which point a planet from a given initial orbit is swallowed. 
Figure 12 has been calculated by performing this calculation for a series of $3 M_{J}$ planet:4 4 , using the evolutionary histories for metal-rich stars of mass $1.5 M_{\odot}$ and $2.5 M_{\odot}$, using the Padova models (Girardi et al. 2000). We show the results as a function of stellar radius, as a proxy for age. Also shown (dotted line) is the criterion indicating the orbital period at the surface of the star. This is the line used by Johnson et al., and it indeed reaches orbital periods of several days only after the star has evolved significantly. The criterion derived from tidal evolution is somewhat stricter, suggesting that orbital periods out as far as 8 days are denuded by the time the star reaches the subgiant stage, and periods out to $\sim 40$ days are removed by the time the star reaches a radius of $10 R_{\odot}$. Thus, any true hot Jupiters are removed rapidly, although it remains to be seen whether the remaining gap between 8 and 200 days is real or simply the result of low statistics.

After submission of this paper, Johnson et al. (2010) announced the discovery of HD102956b, a planet in a 6.5 day orbit around an A subgiant. This system supports the latter answer, and lies on the edge of the tidal survival boundary, as shown in Figure 12 .

\subsection{Spin of the Host Stars}

The above calculations have assumed that the host stars are spinning slowly, consistent with what is observed for the majority of systems. If the star was spinning sufficiently rapidly, the tidal transfer of angular momentum from stellar spin to planetary orbit could potentially drive the planet outwards (e.g. Dobbs-Dixon, Lin \& Mardling 2004). It has been noted that many of the massive planet hosts discussed in $\S 4.3$ are rotating more rapidly than the average for the sample as a whole, so we have investigated the effects of stellar spin on our calibration. In each case we investigated a range of initial stellar spins and repeated the calculation with our original and final values of stellar dissipation. No appreciable change in the period-eccentricity relation was found. As an illustration, consider the case of the HAT-P-2 system that was used to finalise the calibration in $\S$ 4.4.2. This is also the only one of the systems in which the stellar spin is actually faster than the orbital spin, which would reverse the sign of the tide. We repeated our tidal evolution calculations for the same distributions in period and eccentricity, but now also sampling uniformly an initial stellar spin period distribution from 1 to 100 days. One might wonder whether the sign reversal of the stellar-spin dependant term would yield a consistent fit with our original, larger, stellar dissipation rate from $\S 4.2 .7$ Figure 13 shows that this is not the case. In this case we plot final stellar spin against eccentricity, for all periods $<10$ days. We see that it is not possible to match both stellar spin and planetary eccentricity for tidally evolved systems using the stronger dissipation. Ultimately, matching the parameters of the HAT-P-2 system required weakening the stellar tide, irrespective of the stellar spin contribution.

Even if the spin of the star is not dynamically important, it is possible that conservation of

\footnotetext{
${ }^{4}$ Following the claims of Bowler et al. (2010) that the average mass of planets around these stars is higher.
} 
angular momentum would spin the star up, if it drags a planet inwards through tidal coupling. It has been suggested (Pont 2009) that several observed host stars are rotating anomalously rapidly, and that this may indicate a non-negligible influence of tides on the host star itself. Within the context of our model calibration, none of the proposed systems (HD189733, COROT-2b, HAT-P-2b and XO-3b) exhibits a sufficiently strong coupling to spin the stars up to significant levels. The more recently discovered system, WASP-18b, offers perhaps the best chance of observing stellar spin-up, as it is a massive planet in a very short period orbit (Hellier et al. 2009). However, even this system, within the context of stellar solid-body rotation, fails, by an order of magnitude, to spin the star up to the observed rotation period of 5.6 days (assuming a system lifetime of 1.5 Gyr). Thus, we do not expect the dynamics of the previous sections to be significantly affected by stellar rotation. It is possible, however, that the observed rotation period represents the spin-up of the surface convection zone only. WASP-18b is an F-star and thus it is quite possible that the inspiral spun-up the surface convection zone while leaving the interior less rapidly rotating.

The best system for observing outward migration driven by stellar spin is WASP-33b (Collier Cameron et al. 2010). The estimated stellar rotation period is 0.81 days, and the orbital period is 1.2 days. This is sufficiently close that the tidal coupling is strong enough to be important even with the calibration of $\S 4.4 .2$. Figure 14 shows the expected orbital evolution of the system, assuming the surface rotation represents the bulk solid body rotation of the star. We see that the planet is eventually driven out to orbital periods $\sim 2$ days. The effect gets rapidly weaker with distance, and planets with orbital periods $>3$ days experience little change.

\subsection{Internal Structure and Microphysical Dissipation}

We have calibrated the planetary and stellar dissipation using a single bulk constant, within the context of the equilibrium tide model. It is clearly of some interest to understand how the final value compares to more detailed models for the true microphysical dissipation. The simplest microphysical model is dissipation of the equilibrium tide due to the turbulence in the convection zone. In appendix $\mathrm{A}$ we use the formalism of Eggleton et al to relate the bulk $\bar{\sigma}_{p}$ and $\bar{\sigma}_{*}$ to the internal dissipative processes, using our models for planetary and stellar structure.

For the planets, we find that the amount of dissipation required by our calibration is several orders of magnitude lower than that obtained if one adopts a simple local turbulent viscosity based on convective velocities and scale heights. This is encouraging, because the largest eddy overturn times are of the order of years, and so it is not clear how strong a coupling is likely to occur to forcing periods on the order of days. Indeed, there are several proposed prescriptions regarding how inefficient such a coupling is. Zahn (1989) proposes that the strength of the turbulent viscosity is reduced by a linear factor in the period, while Goldreich \& Nicholson (1977) propose a quadratic scaling. Our calculations in appendix $\mathrm{A}$ favour a value intermediate between these prescriptions. This is perhaps not surprising in the light of recent studies by Penev, Barranco \& Sasselov (2009), who have performed simulations of forced anelastic convection. They find rates of dissipation that 
scale similar to the Zahn prescription when the forcing period is not too different from the eddy overturn times, but faster losses of efficiency as the forcing frequency becomes significantly faster. They suggest that the ultimate root of this behaviour is that the turbulence deviates from the Kolmogorov form used to derive the above scalings. While our results clearly cannot shed any further light at this level of detail, it is encouraging that the order of magnitude values are within range of what one might naturally expect.

Our estimate of the stellar dissipation is not as sensitive to the various scalings, as the eddy overturn periods are of order days in this case, and thus should couple well to the observed properties. The effective calibration is a factor $\sim 10$ lower than what we infer from the simple model estimate, so that it is consistent with a slightly inefficient dissipation of the nominal equilibrium tide. The fact that this equilibrium tide value is broadly consistent with our calibration based on planets but not based on stars finds broad support in studies such as those of Ogilvie \& Lin (2007). The essential difference is that planet host stars do not have spins synchronised to the orbital period, while stars in close, equal-mass binaries do. In the latter case, Ogilvie \& Lin show that the equivalence of forcing period and rotation period allows for the excitation of inertial modes (an example of the 'dynamical tide'), which can substantially enhance the rate of tidal dissipation. Similar modes are not excited by planets because the host stars rotate much slower than the orbital period. Excitation of modes in the radiative core (Goodman \& Dickson 1998; Witte \& Savonije 2002; Ogilvie \& Lin 2007; Barker \& Ogilvie 2010) may also contribute an enhanced dissipation the case of planets, but the extent to which they do depends on the uncertain non-linear dissipation of waves that propagate to the center (in the limit of low dissipation, the excitations result in global modes with specific resonant frequencies, which do not generate a broad-band tidal response).

\section{Conclusions}

We have used the current sample of observed exoplanets to calibrate a model for tidal evolution based on that of Eggleton, Kiseleva \& Hut (1998). This formalism has several advantages over those used in many other studies, in that it can be used to treat systems with large eccentricities, and

offers a specific model for the frequency dependence of the tidal dissipation. Our calibration makes use of the distribution of planets and stars in terms of period, eccentricity and mass. Our principal conclusions are

- We can successfully calibrate the model using the distribution of planets. By considering the variation of eccentricity amongst planets of different mass, we are able to simultaneously constrain dissipation in both planets and the main sequence host stars. We find that the calibration requires a level of dissipation in the host star that is inconsistent with the circularisation period of stars in equal-mass main sequence binaries. This agrees with prior studies which find that the generation and dissipation of inertial waves in this latter class of system may lead to enhanced dissipation relative to non-synchronous systems which characterise the 
exoplanet host sample.

- With this calibration we are unable to reproduce prior claims that the bloated radii of some hot Jupiters are the result of recent tidal circularisation and dissipation. This disagreement is not only the result of our calibration, but also due to the fact that our model treats high eccentricity systems with greater fidelity than many previous studies.

- Our calibration of stellar dissipation is consistent with the existence and survival of recently discovered planets with orbital periods $\sim 1$ day or less. We show that planets in this period regime do experience significant tidal evolution on the timescale of Gigayears, but are expected to survive for the lifetimes of their observed hosts.

- We find that this level of tidal inspiral may also explain the lack of planets in close binaries observed around A-type subgiants. As the stellar radius increases, the evolving star exerts a stronger tidal force and drags planets inwards, to the extent that planets as far out as $\sim 8$ days may be swallowed by the time the stars evolve to the point at which they are now observed.

- We have compared our model to simple estimates of turbulent dissipation of the equilibrium tide. Our results suggest a value that lies somewhere between the two proposed scalings for the efficiency of dissipation as a function of forcing frequency.

These results are encouraging in the sense that it is possible to describe the global distribution of exoplanets within a simple tidal evolution model. However, we must also recognise that the equilibrium tide model used is amongst the simplest possible descriptions of a complex physics problem. Given the success of the simple model, it may be of future interest to follow this study with a similar study based on a more complex microphysical description.

The author would like to thank Phil Armitage, Kristen Menou, David Spiegel, Jeremy Leconte and an anonymous referee for comments on the manuscript, and the participants in the Kavli

Institute for Theoretical Physics workshop on Extrasolar Planets for stimulating the beginnings of this project.

\section{REFERENCES}

Anderson, D. R. et al., 2010, ApJ 709, 159

Bakos, G. et al., 2007, ApJ, 670, 826

Barker, A. J. \& Ogilvie, G. I., 2010, arXiv:1001.4009

Barman, T. S., Hauschildt, P. H. \& Allard, F., 2005, ApJ, 632, 1132

Baraffe, I., Chabrier, G., Allard, F. \& Hauschildt, P. H., 1998, A\&A, 337, 403 
Baraffe, I., Chabrier, G., Barman, T., Allard, F. \& Hauschildt, P., 2003, A\&A, 407, 701

Batygin, K., Bodenheimer, P. \& Laughlin, G., 2009, ApJ, 704, L49

Bodenheimer, P. R., Lin, D. N. C., \& Mardling, R. A., 2001, ApJ, 548, 466

Bowler, B. P., et al., 2010, ApJ, 709, 396

Burrows, A., Guillot, T., Hubbard, W. B., Marley, M. S., Saumon,D., Lunine, J. I. \& Sudarsky, D., 2000, ApJ, 534, L97

Butler, R. P., Vogt, S., Marcy, G., Fischer, D., Henry, G. \& Apps, K., 2000, ApJ, 545, 504

Campo, C. et al., 2010, arXiv:1003.2763

Collier Cameron, A., et al., 2010, arXiv:1004.4551

Da Silva, R. et al., 2005, A\&A, 446, 717

Dobbs-Dixon, I., Lin, D. N. C. \& Mardling, R. A., 2004, ApJ, 610, 464

Dunham, E., et al., 2010, ApJ, 713, L136

Eggleton, P., Kiseleva, L. \& Hut, P., 1998, ApJ, 499, 853

Girardi, L., Bresson, A., Bertelli, C. \& Chiosi, C., 2000, A\&AS, 141, 371

Goldreich, P. \& Nicholson, P. D., 1977, Icarus, 30, 301

Goldreich, P. \& Soter, S., 1966, Icarus, 5, 375

Goodman, J. \& Dickson, E., 1998, ApJ, 507, 938

Greenberg, R., 2009, ApJ, 698, L42

Guillot, T., Burrows, A., Hubbard, W. B., Lunine, J. I. \& Saumon, D., 1996, ApJ, 459, L35

Hansen, B. \& Barman, T., 2007, ApJ, 671, 861

Hebb, L. et al., 2009, ApJ, 693, 1920

Hebb, L. et al., 2010, ApJ, 708, 224

Hellier, C., et al., 2009, Nature, 460, 1098

Husnoo, N., et al., 2010, arXiv:1004.1809

Hut, P, 1981, A\&A, 99, 126

Ibgui, L., Burrows, A. \& Spiegel, D. S., 2010, ApJ, 713, 751

Jackson, B., Greenberg, R. \& Barnes, R., 2008a, ApJ, 678, 1396

Jackson, B., Greenberg, R. \& Barnes, R., 2008b, ApJ, 681, 1631

Jackson, B., Barnes, R. \& Greenberg, R., 2009, ApJ, 698, 1357

Johns-Krull, C. et al., 2008, ApJ, 677, 657

Johnson, J., Marcy, G., Fischer, D., Henry, G., Wright, J., Isaacson, H. \& McCarthy, C., 2006, ApJ, 652, 1724 
Johnson, J. et al., 2007, ApJ, 665, 785

Joshi, Y. et al., 2009, MNRAS, 392, 1532

Lainey, V., Arlot, J.-E., Karatekin, Ö \& van Hoolst, T., 2009, Nature, 459, 957

Leconte, J., Chabrier, G., Baraffe, I. \& Levrard, H., 2010, arXiv:1004.0463

Lopez-Morales, M., Coughlin, J. L., Sing, D. K., Burrows, A., Apai, D., Rogers, J. C. \& Spiegel, D. S., 2009, arXiv:0912.2359

Lovis, C. \& Mayor, M., 2007, A\&A, 472, 657

Marcy, G. W., Butler, R. P., Williams, E., Bildsten, L., Graham, J. R., Ghez, A. M. \& Jernigan, J. G., 1997, ApJ, 481, 926

Mardling, R., 2007, MNRAS, 382, 1768

Matsumura, S., Takeda, G. \& Rasio, F. A., 2008, ApJ, 686, L29

Meibom, S. \& Mathieu, R. D., 2005, ApJ, 620, 970

Miller, N., Fortney, J. \& Jackson, B., 2009, ApJ, 702, 1413

Niedzielski, A. et al., 2009, ApJ, 707, 768

Paxton, B., 2004, PASP, 116, 699

Peale, S. J., 1999, ARA\&A, 37, 533

Peale, S. J. \& Lee, M. H., 2002, Science, 298, 593

Penev, K., Barranco, J. \& Sasselov, D., 2009, ApJ, 705, 285

Pont, F., 2009, MNRAS, 396, 1789

Rasio, F. A., Tout, C. A., Lubow, S. H. \& Livio, M., 1996, ApJ, 470, 1187

Rauer, H. et al., 2009, A\&A, 506, 281

Sato, B., et al., 2008, PASJ, 60, 539

Winn, J. et al., 2008, ApJ, 683, 1076

Witte, M. G. \& Savonije, G. J., 2002, A\&A, 386, 222

Zahn, J.-P. \& Bouchet, L., 1989, A\&A 223, 112

Zahn, J.-P, 1989, A\&A, 220, 112 
Fig. 1. - The curves show the stellar radius evolution from the models of Baraffe et al. (1998), for masses of $0.8,1.0$ and $1.2 M_{\odot}$. Although the $1.2 M_{\odot}$ star is only about $60 \%$ larger than the $0.8 M_{\odot}$ star over most of the age range, this can amount to a factor of 100 when raised to the tenth power. The temporal evolution of a given mass star has an even stronger effect.

Fig. 2.- All three curves have been calculated for a $1.41 M_{J}$ planet, with an irradiation appropriate to that of the WASP-12b system. The solid curve assumes no extra internal heating due to tides. It can be compared with the curves in Figure 11 of Miller et al. (2009) and Figure 2 of Ibgui, Spiegel $\&$ Burrows (2009). The dotted and dashed curves represent attempts to match the figures of those respective papers by parameterising our models to yield the same value of $Q_{p}$ and $Q_{*}$ at the current semi-major axis of WASP-12. We also incorporate an eccentricity floor of 0.05 for the dotted line, which yields the same runaway heating as used by Miller et al. Such an eccentricity was initially claimed for WASP-12, but subsequent observations seem to belie this, so the approximation may not be valid.

Fig. 3.- The points indicate the circularisation period as a function of age for different stellar populations, from Meibom \& Mathieu (2005). The solid line is the best-fit version of our model, including pre-main sequence evolution. For comparison, the dotted line is what we obtain with the best fit value of $\tau_{p}$ for the field population of the local solar neighbourhood, but assuming a fixed radius of $1 R_{\odot}$. We see that this strongly underpredicts the circularisation of early-type binaries.

Fig. 4.- The top panel shows the eccentricity-period relation for the 0.15 Gyr old open cluster M35 (Meibom \& Mathieu 2005). The lower panel shows the eccentricity-period relation for the sample of exoplanets with masses between $0.3 M_{J}$ and $3 M_{J}$, that orbit stars with masses between $0.7 M_{\odot}$ and $1.5 M_{\odot}$. In each panel, we show the best-fit circularisation function for that sample.

Fig. 5.- The solid points show the eccentricity-period relation for Jovian-mass exoplanets. The small dots indicate a model population, drawn from the long-period eccentricity distribution and then tidally evolved for $3.0 \mathrm{Gyr}$, with our best fit values for $\bar{\sigma}_{p}$ and $\bar{\sigma}_{*}$. The model traces the envelope of the observations very well. The thinning out of model points above $e \sim 0.5$ is the result of the original distribution, which had a mean of 0.2 and a dispersion of 0.25 , as dictated by the long period objects.

Fig. 6. - The lower panel masses between $0.3 M_{J}$ and $3 M_{J}$, that orbit stars with masses between $0.7 M_{\odot}$ and $1.5 M_{\odot}$. The upper panel shows the equivalent sample of planets with masses above $3 M_{J}$. The more massive planets appear more eccentric.

Fig. 7.- The top two panels show the comparison between observations and model realisations for the original parameterisation of tidal dissipation in $\S$ 4.2.7 for the cases of the massive planet systems WASP-14b and XO-3. In the bottom two panels, we show the same comparisons, but now with the modified calibration of $\S 4.4 .2$, tuned to fit the most extreme massive system HAT-P-2b. 
Table 1. Tidal circularisation periods for solar mass binaries of various ages.

\begin{tabular}{lccc}
\hline \hline \multicolumn{1}{c}{ Population } & $\log t_{9}$ & $P^{\prime}($ days $)$ & $10^{5} \bar{\sigma}_{*}$ \\
\hline PMS binaries & -2.5 & $7.1_{-1.2}^{+1.2}$ & $6 \pm 2$ \\
Pleiades & -1.0 & $7.2_{-1.9}^{+1.8}$ & $3.1 \pm 2$ \\
M35 & -0.8 & $10.2_{-1.5}^{+1.0}$ & $9.7 \pm 4.8$ \\
Hyades/Praesepe & -0.2 & $3.2_{-1.2}^{+1.2}$ & $\ldots$ \\
M67 & 0.6 & $12.1_{-1.5}^{+1.0}$ & $7.0 \pm 3.0$ \\
NGC188 & 0.8 & $14.5_{-2.2}^{+1.4}$ & $9.7 \pm 3.8$ \\
Field & 0.95 & $10.3_{-3.1}^{+1.5}$ & $0.8 \pm 0.6$ \\
Halo & 1.0 & $15.6_{-3.2}^{+2.3}$ & $3.6 \pm 2.0$ \\
\hline
\end{tabular}

Table 2. Tidal Inspiral Times for Close Planets

\begin{tabular}{lccccc}
\hline \hline \multicolumn{1}{c}{ Planet } & $M_{p}\left(M_{J}\right)$ & $\mathrm{R}_{*} / \mathrm{R}_{\odot}$ & $\mathrm{a}(\mathrm{AU})$ & $\mathrm{T}_{i n}(\mathrm{Gyr})$ & Age $(\mathrm{Gyr})$ \\
\hline WASP-19b & $1.15 \pm 0.08$ & $0.93 \pm 0.05$ & 0.0164 & $3.0 \pm 0.2$ & 0.6 \\
COROT-7b & $0.0151 \pm 0.0025$ & $0.93 \pm 0.03$ & 0.0172 & $336 \pm 56$ & 1.5 \\
WASP-18b & $10.4 \pm 0.4$ & $1.23 \pm 0.05$ & 0.02047 & $0.12 \pm 0.005$ & $0.63_{-0.53}^{+0.95}$ \\
OGLE-TR-56 & $1.29 \pm 0.12$ & $1.32 \pm 0.06$ & 0.0225 & $1.1 \pm 0.1$ & $>2$ \\
TrES-3 & $1.92 \pm 0.23$ & $0.813_{-0.027}^{+0.012}$ & 0.0226 & $82 \pm 10$ & $\ldots$ \\
OGLE-TR-113 & $1.32 \pm 0.19$ & $0.77 \pm 0.02$ & 0.0229 & $249 \pm 36$ & $>0.7$ \\
WASP-12b & $1.41 \pm 0.1$ & $1.57 \pm 0.07$ & 0.0229 & $0.24 \pm 0.02$ & $\ldots$ \\
WASP-4b & $1.12 \pm 0.08$ & $1.15 \pm 0.28$ & 0.023 & $5.5 \pm 1.3$ & $\ldots$ \\
COROT-1b & $1.03 \pm 0.12$ & $1.11 \pm 0.05$ & 0.0254 & $18 \pm 2$ & $\ldots$ \\
WASP-33b & $<4.1$ & $1.44 \pm 0.03$ & 0.0256 & $>0.36$ & 0.25 \\
WASP-5b & $1.64 \pm 0.08$ & $1.08 \pm 0.04$ & 0.0273 & $27 \pm 1$ & $3 \pm 1.4$ \\
COROT-2b & $3.31 \pm 0.16$ & $0.90 \pm 0.02$ & 0.0281 & $108 \pm 5$ & $\ldots$ \\
\hline
\end{tabular}


Fig. 8. - The upper curve shows the evolution of a star-planet with the parameters of the XO-3b system. The solid line shows the first $3 \mathrm{Gyr}$ of tidal evolution using our nominal parameterisation of the equilibrium tide model. The dotted line shows the subsequent evolution of the system. The lower curve shows the same evolution keeping every parameter the same, except for the mass of the planet, which is reduced to $1 M_{J}$. The divergence of these curves shows why the envelope of the period-eccentricity relation is different between planets of different masses.

Fig. 9.- The solid histogram shows the cumulative distribution of planets within $0.04 \mathrm{Au}$ (excluding M star systems) discovered in transit surveys. The short dashed line indicates the distribution expected from our model with the final calibrations, at an age of $3 \mathrm{Gyr}$. The dotted line is for the same model, but with an age of 0.1 Gyr. We see that these trace roughly the correct distribution. The upper curve is the distribution in the model (at $3 \mathrm{Gyr}$ ) if we set $\bar{\sigma}_{*}=0$. Clearly this dramatically overpredicts the number of close planetary systems. The lower dashed curve is recovered if we use the value of $\bar{\sigma}_{*}$ derived from the stellar binaries. This clearly underpredicts the number of close planets. All model curves are weighted by $1 / a$, in order to approximately account for the detectability in a transit survey.

Fig. 10.- The relation between the orbital period of a planet at an age of $10^{8} \mathrm{yrs}\left(P_{8}\right)$ and an age of $10^{9}$ yrs $\left(P_{9}\right)$ indicates that systems with orbital period $<1.5$ days are expected to undergo significant orbital evolution on astrophysical timescales. At the upper end of the plot, we see that the degree of circularisation changes on similar timescales for periods $>2.5$ days.

Fig. 11. - The points show the estimated present day values of $Q_{p}^{\prime}$ and $Q_{*}^{\prime}$ for the known hotJupiter systems, given our calibration of the equilibrium tide model. A few of the outliers are labelled.

Fig. 12.- The solid and dashed lines indicate the critical period for tidal swallowing for $1.5 M_{\odot}$ and $2.5 M_{\odot}$ stars respectively, as a function of the stellar radius. The dotted line indicates the Keplerian orbital period at the surface of the evolving $1.5 M_{\odot}$ star. The filled circles are the observed orbital periods for radial velocity planets around stars in the mass range 1.5-2.5 $M_{\odot}$.

Fig. 13. - The stellar rotation period versus planetary orbit eccentricity is shown for the HAT-P-2 system parameters, after 2 Gyr of tidal evolution, and final orbital periods less than 10 days. The solid point indicates the observed parameters, and the dots show the final state for a variety of initial conditions, assuming the dissipation calibrations of $\S 4.2 .7$. The fact that the simulations do not match the observed value indicates that rapid initial stellar spins cannot stabilise the system in the face of strong stellar tidal dissipation.

Fig. 14. - The solid line indicates the expected orbital evolution of the planet WASP-33b, driven by transfer of angular momentum from the spin of the host star to the planetary orbit. The dashed lines are for other hypothetical systems in which WASP-33b began further out. We see that the effects of tides decrease rapidly with distance. Note we have not included any effects on the stellar rotation due to magnetic braking, mass loss or evolutionary changes in the star. These 
will eventually become important and will likely change the evolutionary direction from outward to inward.

Fig. 15. - The left hand panel shows the evolution of the WASP-12b system and the right hand panel shows the evolution of the WASP-4b system. In both cases the dotted curve traces the semi-major axis evolution of the system, and corresponds to the left axis of the panel. The solid line shows the evolution of the transit radius for the two systems, and corresponds to the right axis of each panel. We see that planetary inflation does occur during tidal circulisation, but that this occurs on timescales $\sim 10^{7}$ years, so that its influence is lost once the planet has evolved for many planetary Kelvin-Helmholtz times, as is the case for these systems.

Fig. 16. - The solid curve shows the evolution of planetary transit radius with semi-major axis during the inspiral of the WASP-12b system shown in the left hand panel Figure 15. The dotted and dashed lines show the evolution of the same system but where we have imposed an artificial lower limit to the eccentricity of the orbit, as labelled. This results in an ongoing dissipation in the planet, which can serve to inflate the planetary radius at late times.

Fig. 17. - The solid curve is the profile of the convective velocity in the planet, given by the left hand axis. The dashed curve is the local eddy turnover time $\tau=\ell_{m} / V_{c}$, where $\ell_{m}$ is taken to be a pressure scale height.

Fig. 18. - The upper panel shows the radial profile of the function $\alpha$, which represents the quadropolar distortion of the planet. We see this is pretty flat over most of the volume. The function $\gamma$ is the weight function in EKH equation (113), which describes how the tidal velocity field couples to the turbulent dissipation. We see this favours the outer parts of the planet. In the lower panel, we show three functions representing the local turbulent dissipation in the planet. The upper curve represents $V_{c} \ell_{m}$, while the middle curve is $V_{c} \ell_{m} T / 2 \tau$, where $\mathrm{T}(=3$ days) is the tidal forcing period, and $\tau$ is the local eddy turnover time. The lower curve is $V_{c} \ell_{m}(T / 2 \pi \tau)^{2}$.

Fig. 19. - The solid curve is the profile of the convective velocity in the outer convection zone of a 5 Gyr old, $1 M_{\odot}$ stars. The dashed curve indicates the eddy turnover time in this model, in units of days. Thus, close-in planets with orbital periods of order days will match these timescales quite well. 


\section{A. Internal Models and Turbulent Dissipation}

In order to relate our bulk dissipation to the underlying microphysics, we need to evaluate the various equations for the quadropolar distortion given by EKH using a background from our planetary models. Figure [17 shows the profile of convective velocity and large eddy turnover time $\tau$ for a $1 M_{J}$ planet at an age of $10^{7}$ years (we choose a young, hot model because this is the age where planets undergo the bulk of their circularisation with our parameterisation). We see that $\tau$ has a value of order months over the bulk of the planet, so that the coupling to the forcing period will not be particularly efficient.

Using the formalism from EKH, we can evaluate the profile of the quadropolar distortion $\alpha(r)$, and the function $\gamma(r)$, which describes how the distortion couples to the microphysical dissipation. Figure 18 shows, in the top panel, the radial profile of these two functions. The distortion itself is relative flat throughout most of the planet (whose density profile is approximately that of an $\mathrm{n}=1$ polytrope), but the function $\gamma$ ascribes a greater weight to the dissipation in the outer half of the planetary volume. In the lower panel of Figure 18 we show the radial profile of the turbulent viscosity $V_{c} \ell_{m}$ (where $\ell_{m}$ is the eddy scale, taken to be a pressure scale height) in three cases. The upper curve is the full viscosity, with no corrections for inefficiency. The two curves below that indicate the estimated local turbulent viscosity with linear and quadratic scalings for the reduction in inefficiency, as suggested by Zahn or Goldreich \& Nicholson respectively. In these cases we have assumed a forcing frequency of 3 days.

Following equation (113) of EKH, one can integrate over the above profiles and infer a bulk viscosity. In the case of a fully efficient coupling, we infer $\bar{\sigma}_{p} \sim 1.9 \times 10^{-4}$, which is $\sim 500$ times too strong. In the case of a linear scaling, $\bar{\sigma}_{p} \sim 1.2 \times 10^{-5}$, which is still a factor of 30 too strong. For a quadratic scaling, $\bar{\sigma}_{p} \sim 9.5 \times 10^{-8}$, which is only a factor of 3 weaker than our nominal calibration. Thus, our inferred bulk dissipation is not very different from what one might expect on the basis of the generally accepted picture of dissipation in turbulent convection.

In the case of the stellar dissipation, Figure 19 shows the convective velocities and eddy turnover times for the outer convection zone of a $5 \mathrm{Gyr}, 1 M_{\odot}$ solar mass, taken from the EZ model (Paxton 2004). Repeating the same exercise for the solar mass star yields $\bar{\sigma}_{*} \sim 7.8 \times 10^{-7}$, which is a factor $\sim 10$ larger than the inferred dissipation. In this case, the efficiency scalings do not make as large a difference, as the turnover times are $\sim$ days, and so the reduction in efficiency only accounts for a factor of 2 change. Thus, the inferred stellar value is also not too different from that expected on the basis of simple turbulent dissipation, with some moderate inefficiency allowed. 


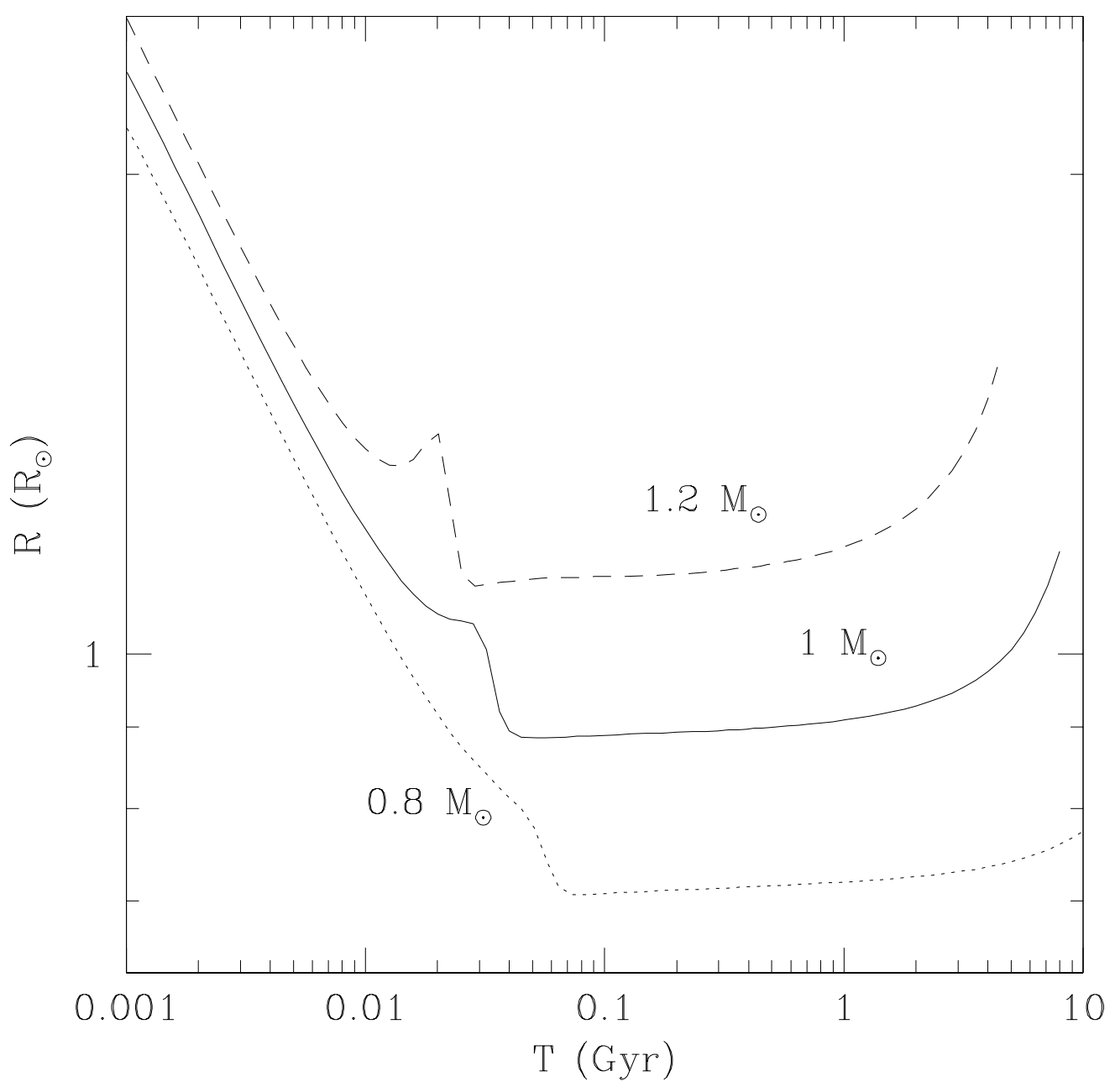




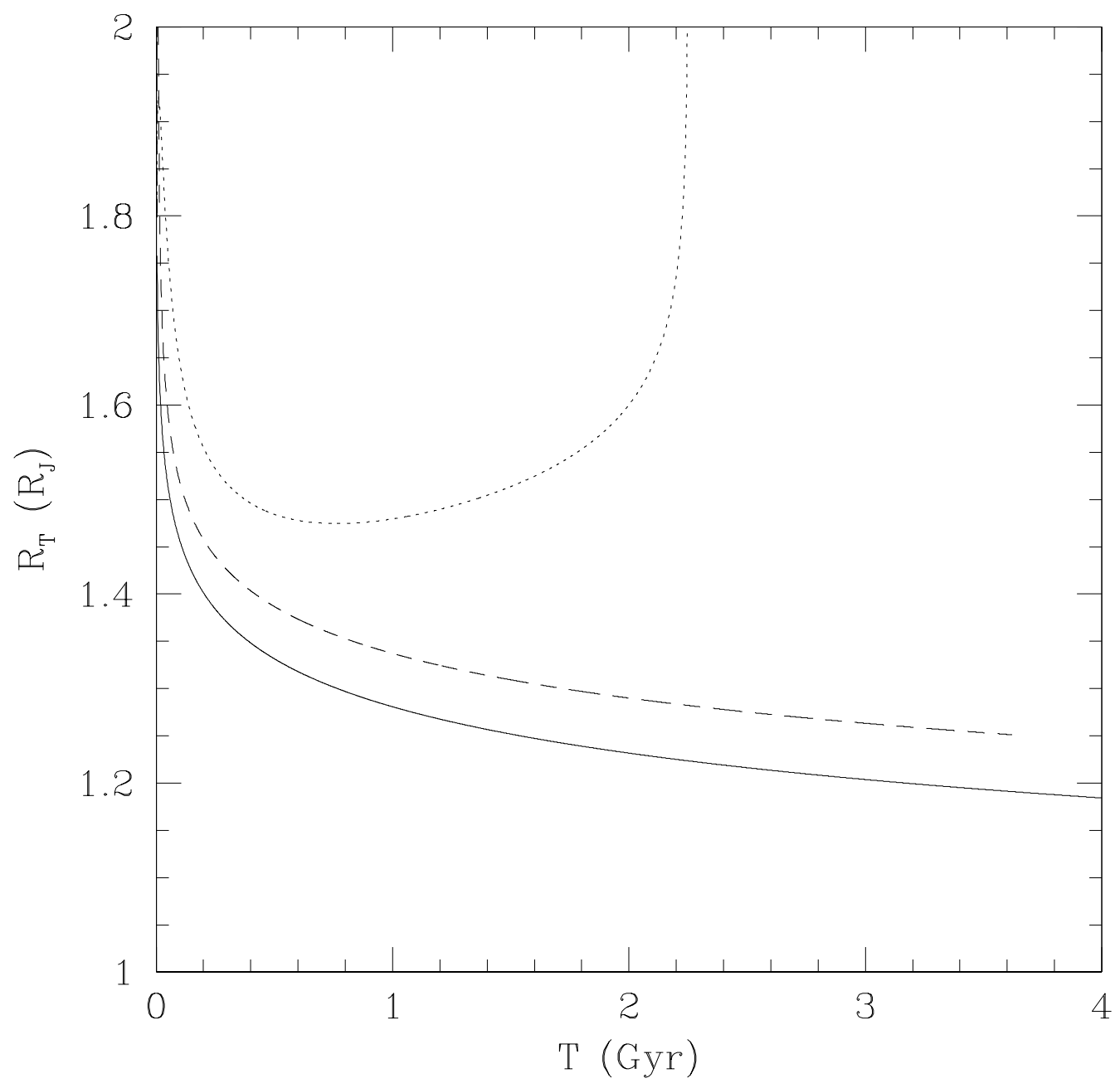




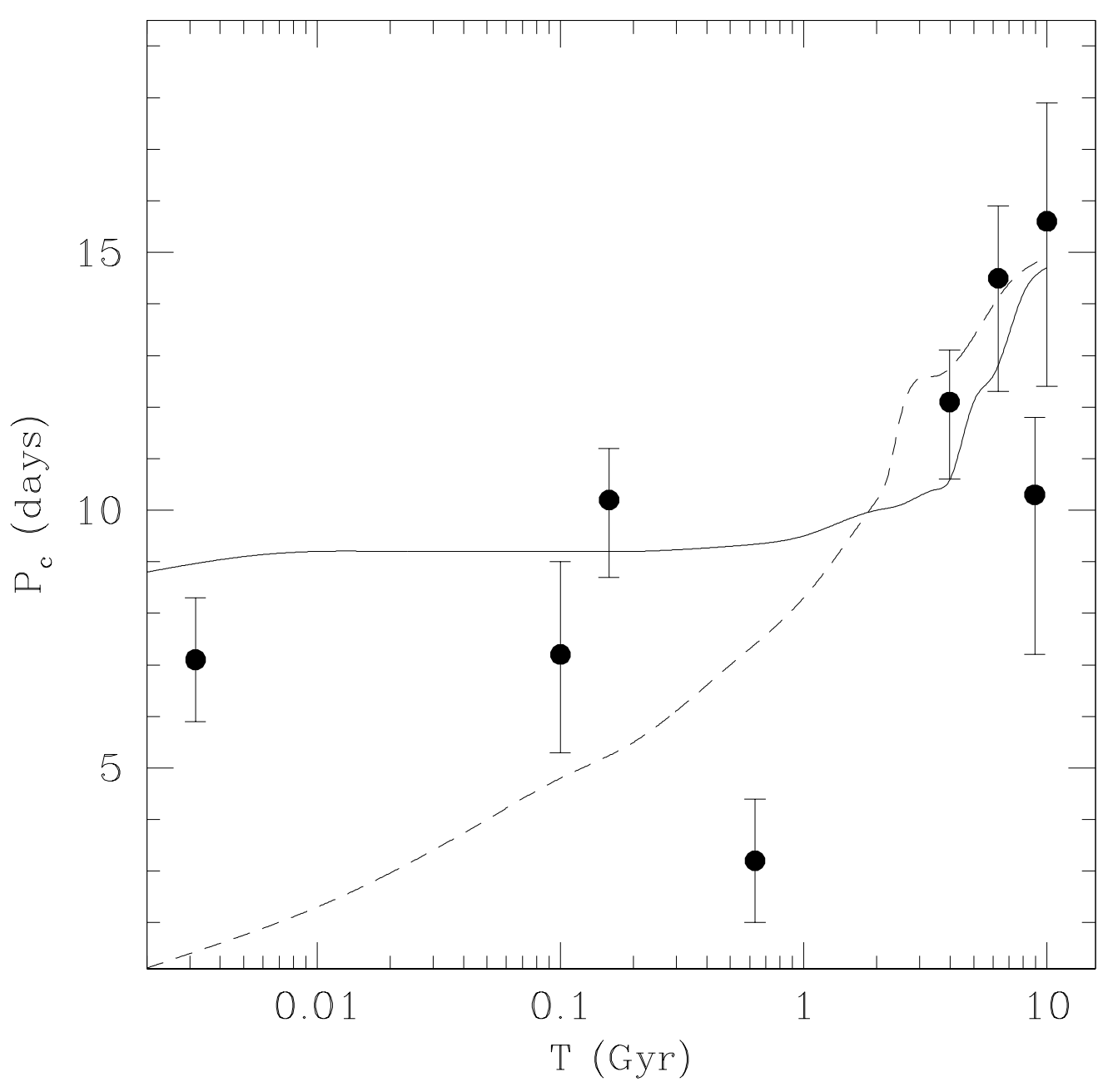



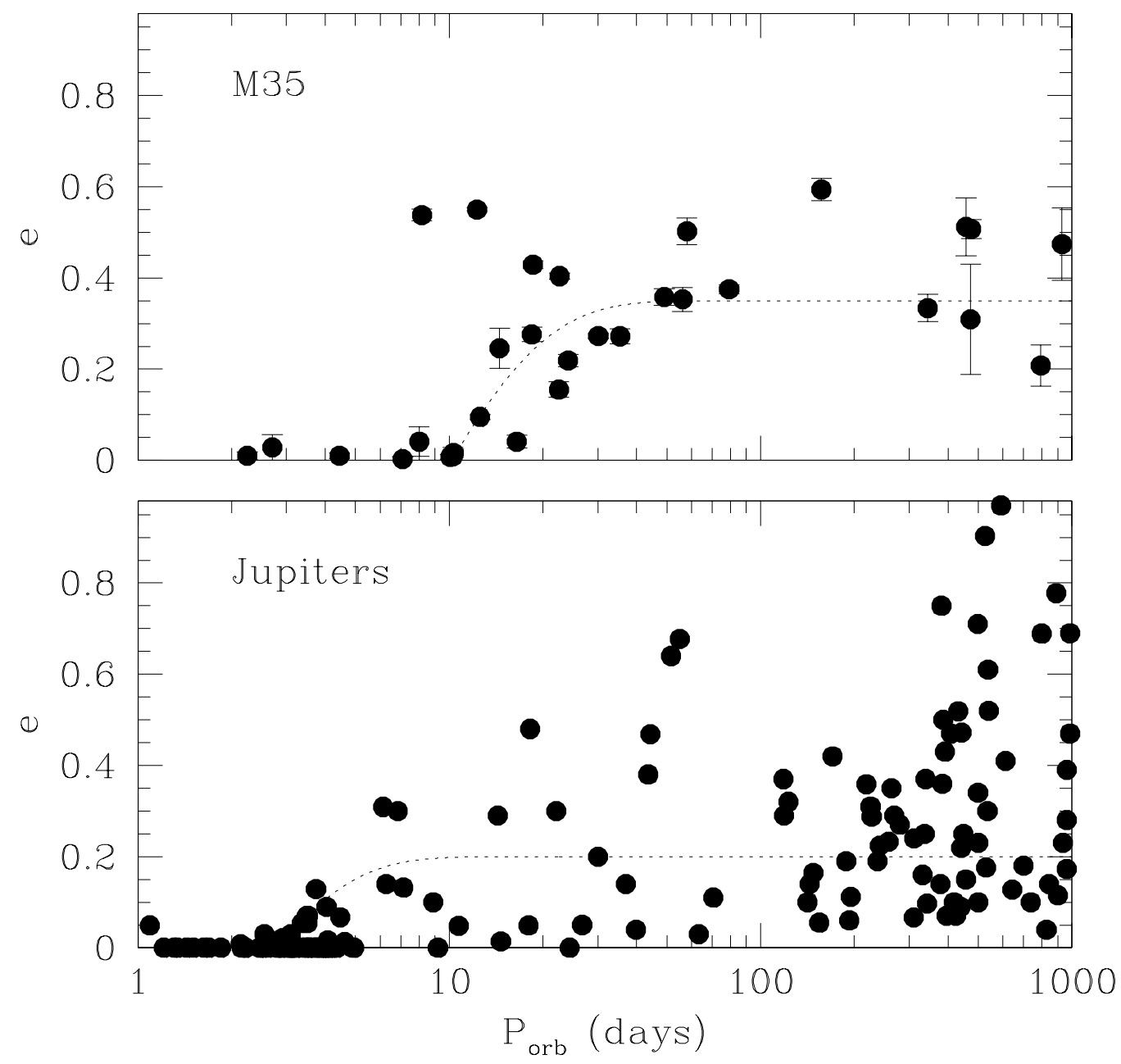


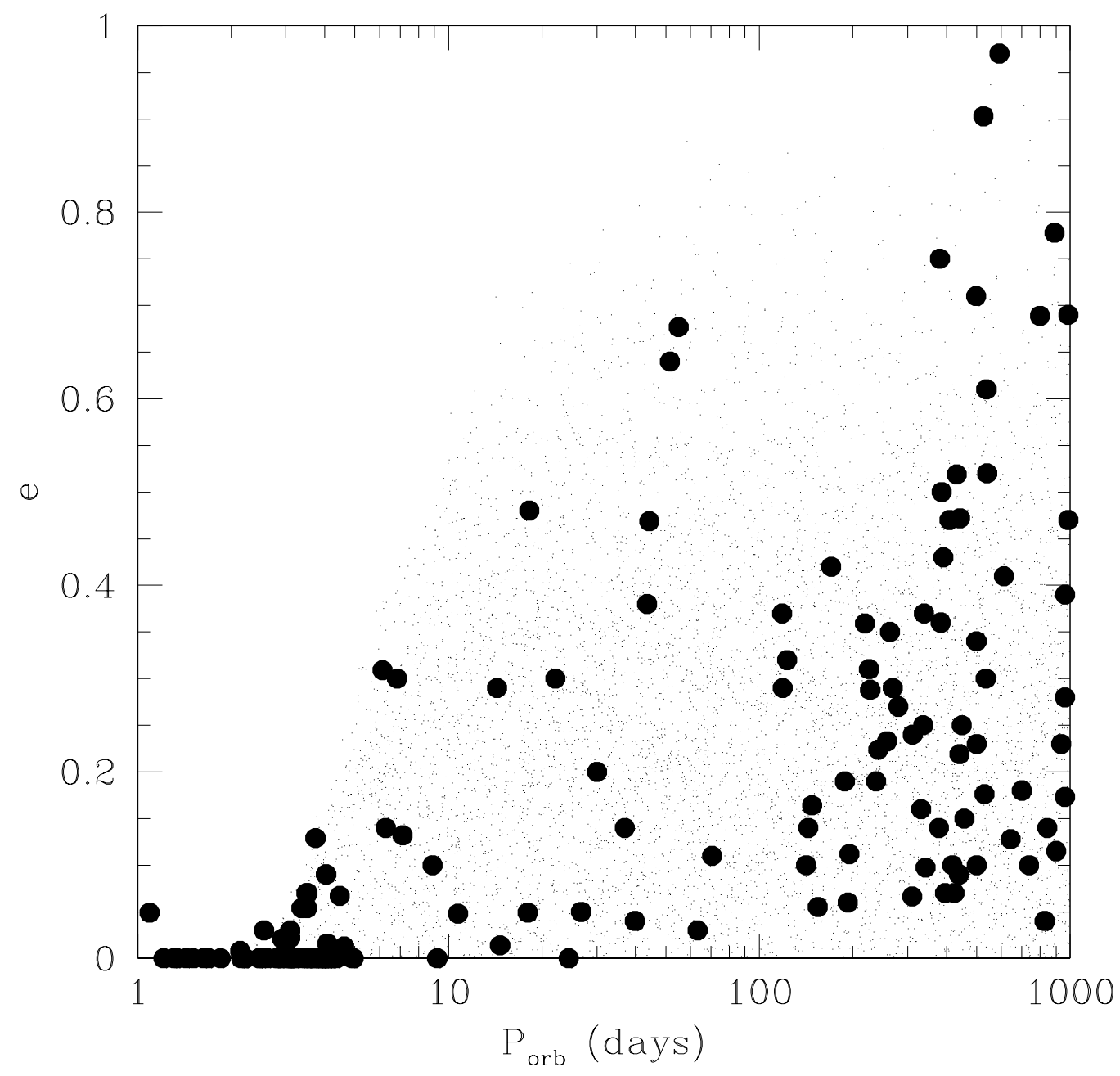



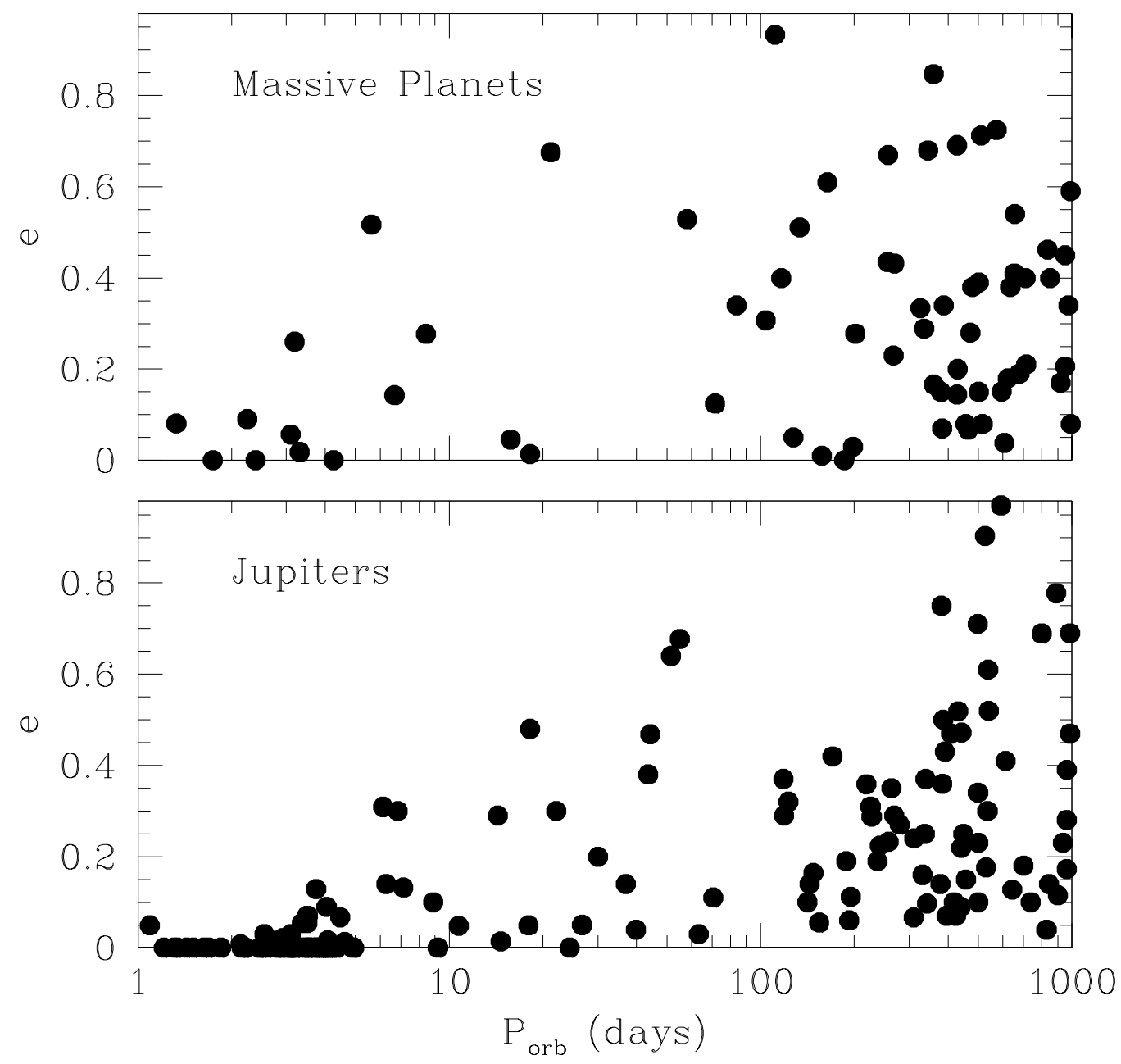


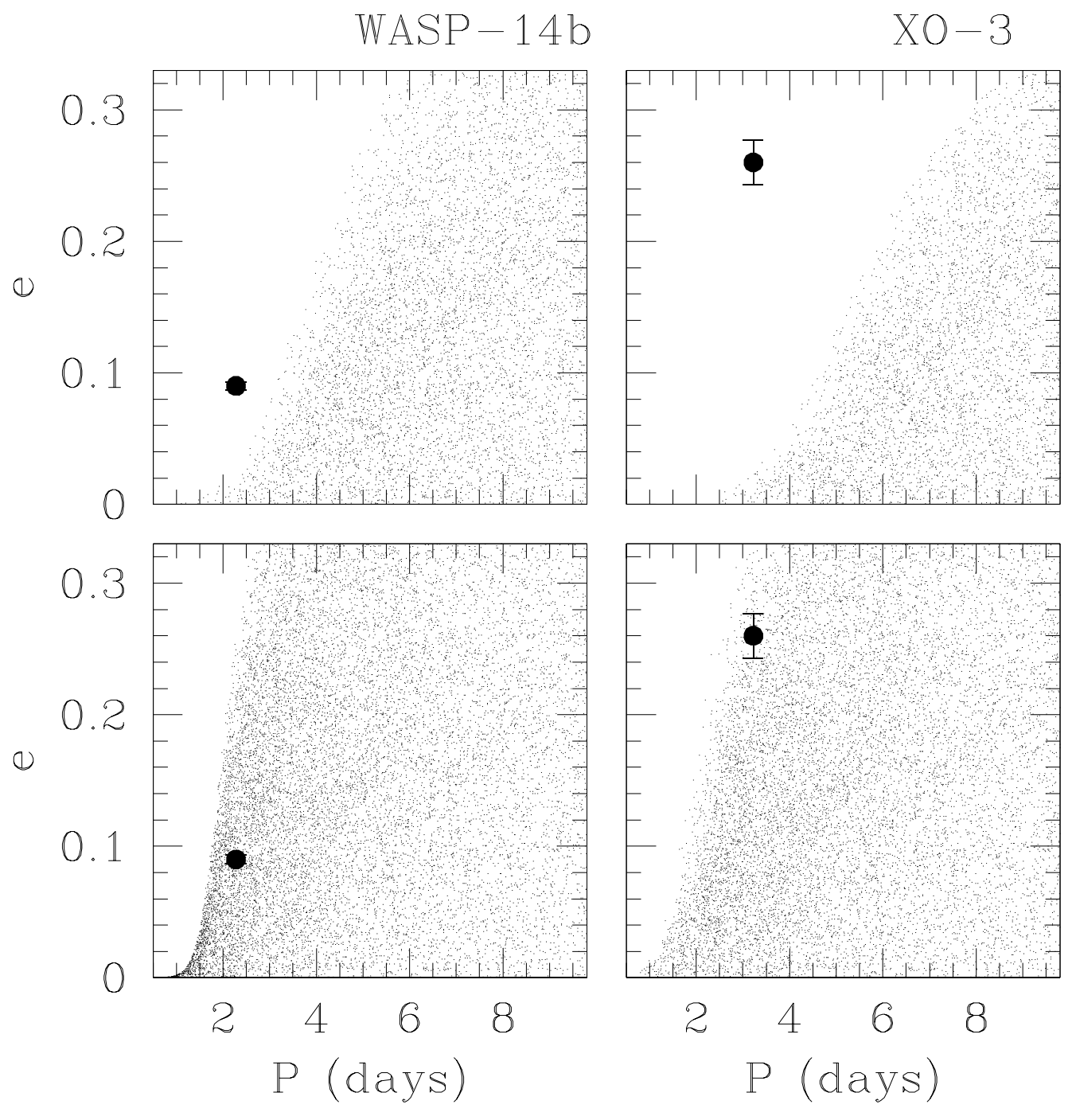




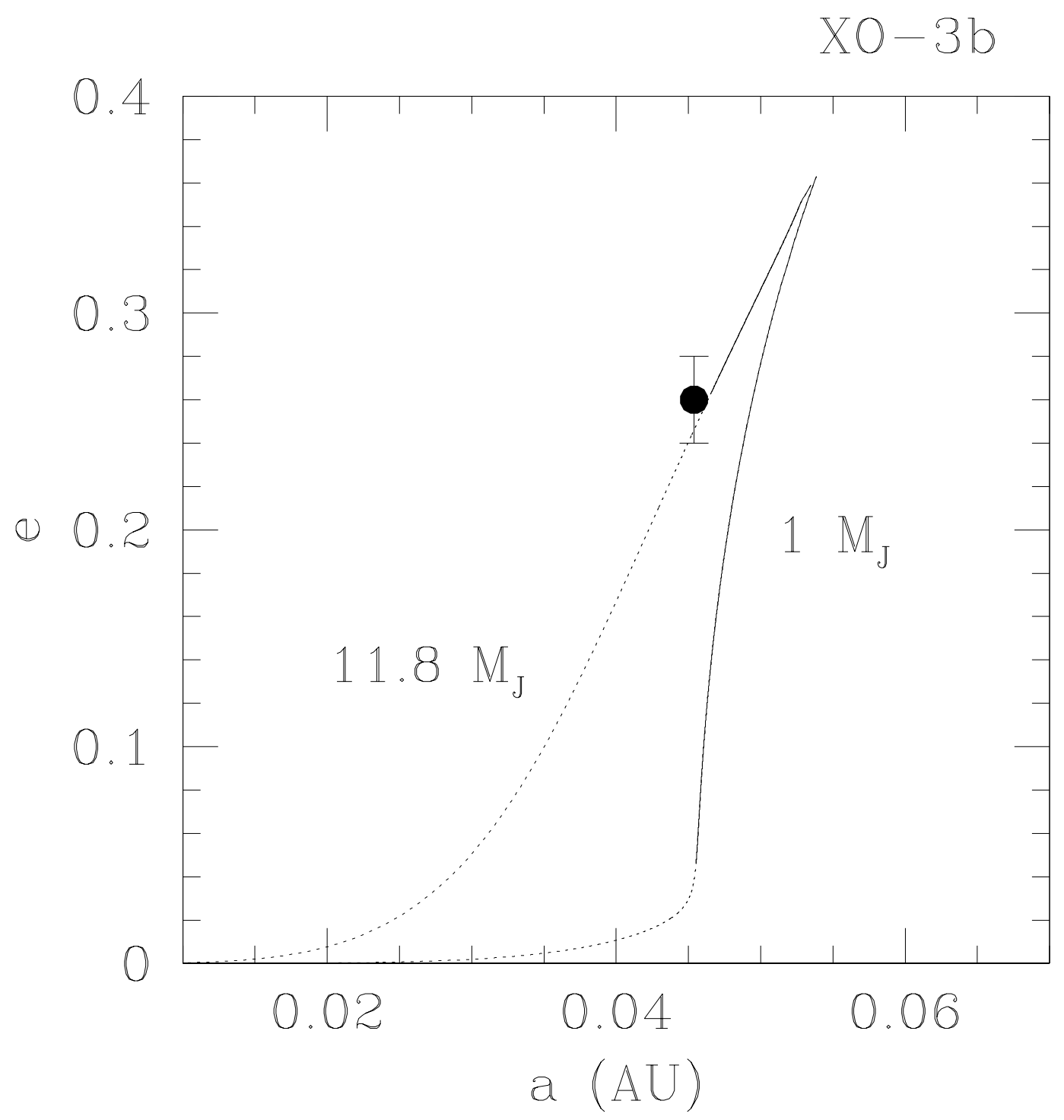




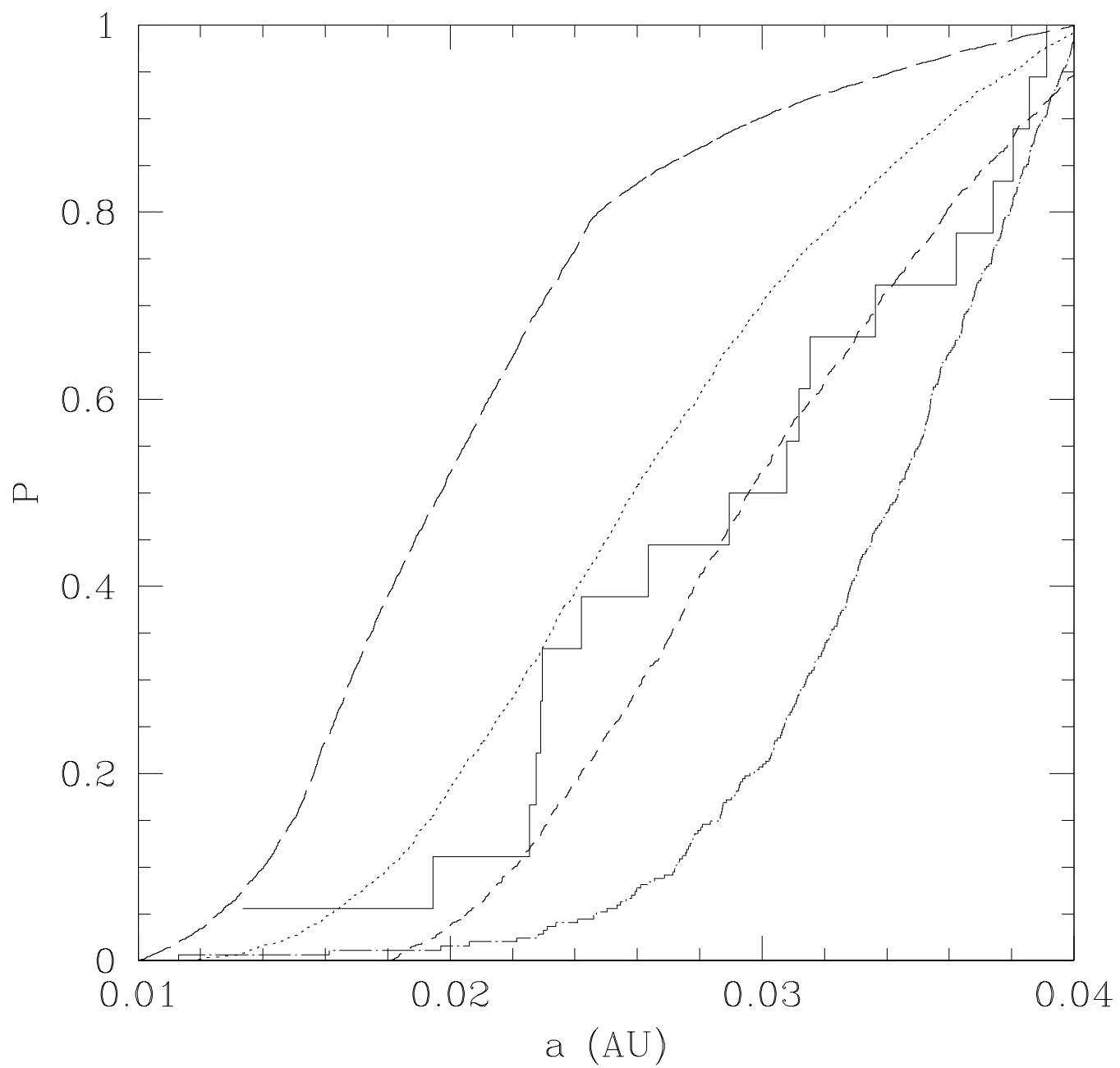




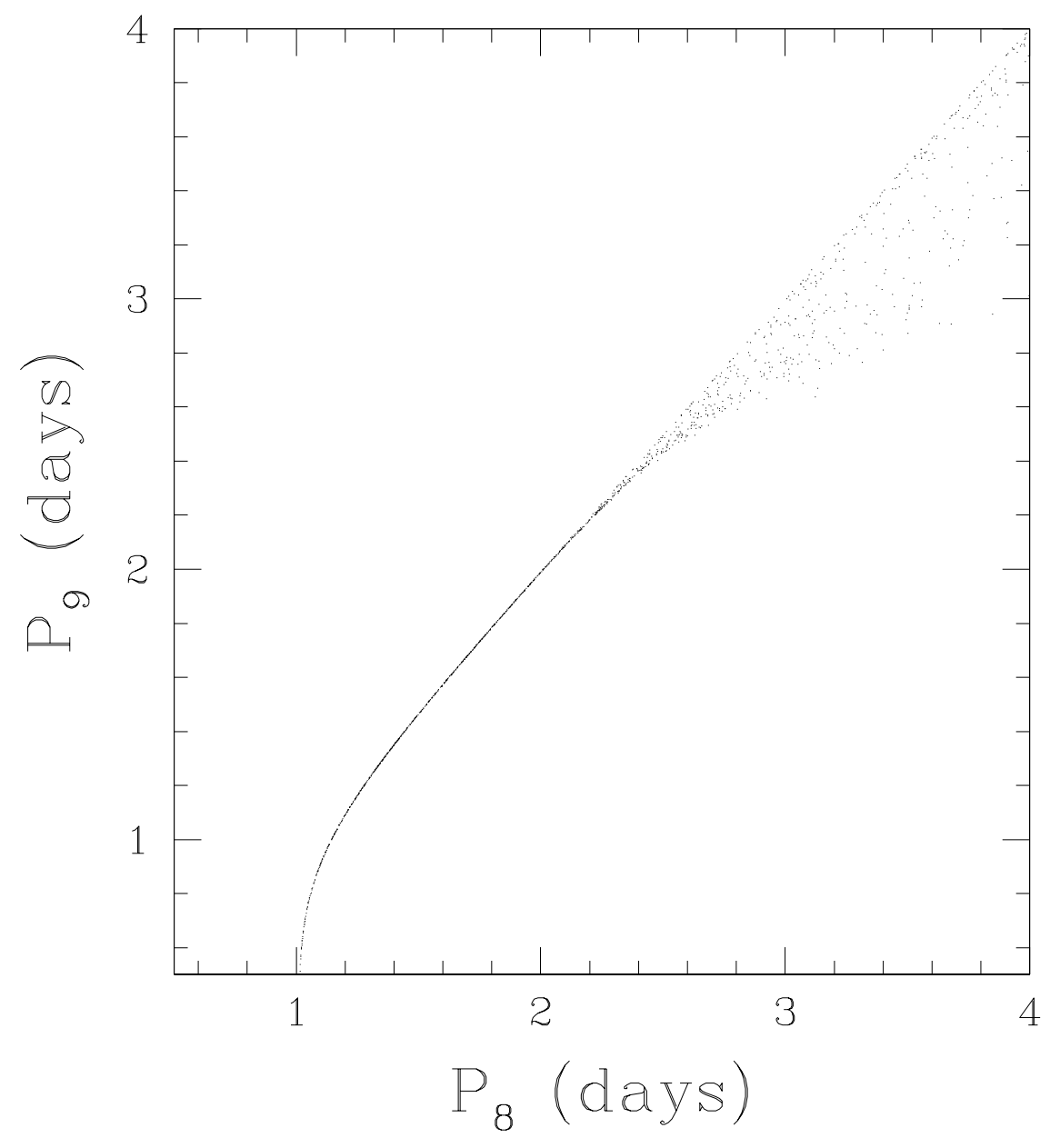




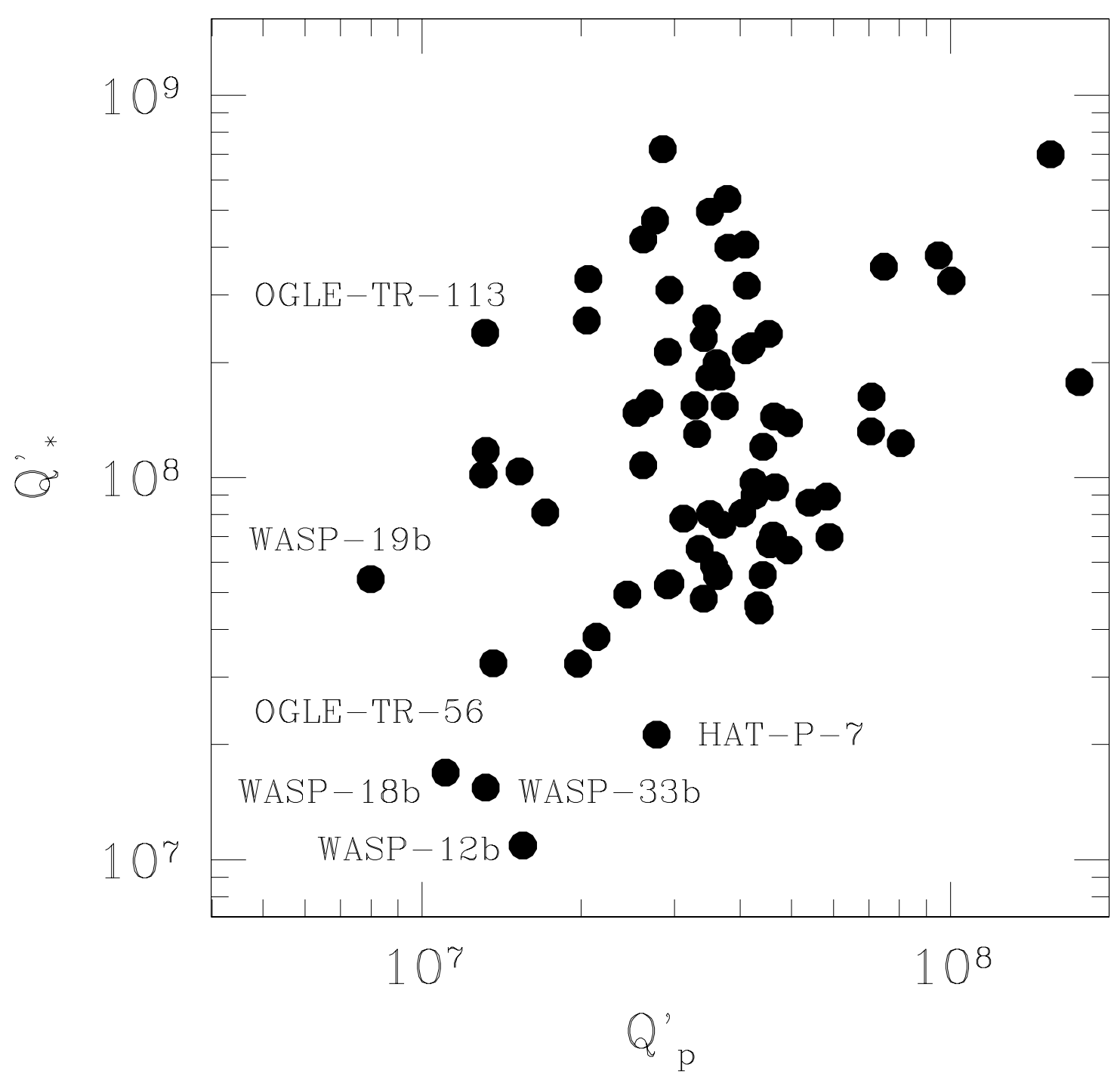




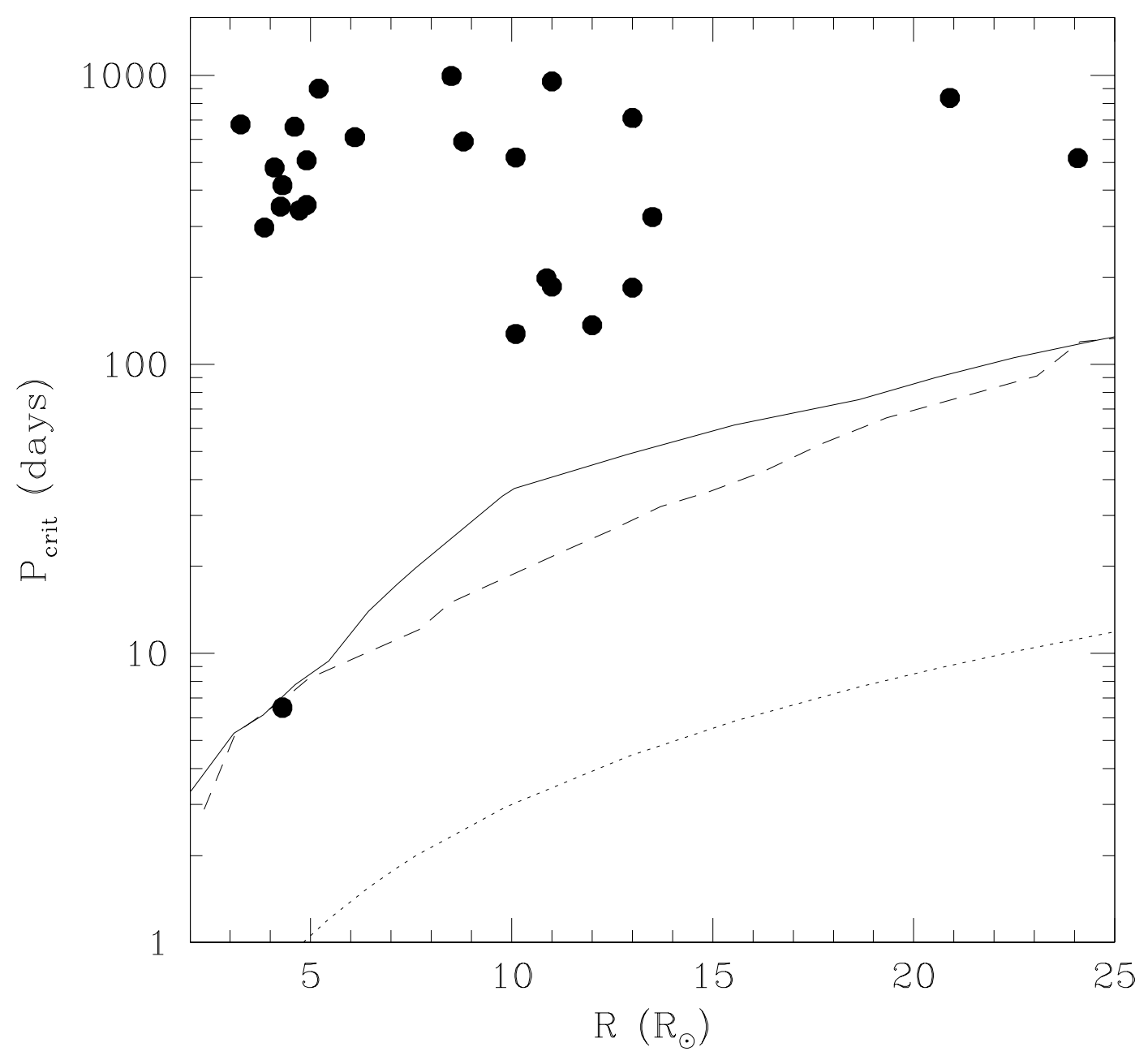




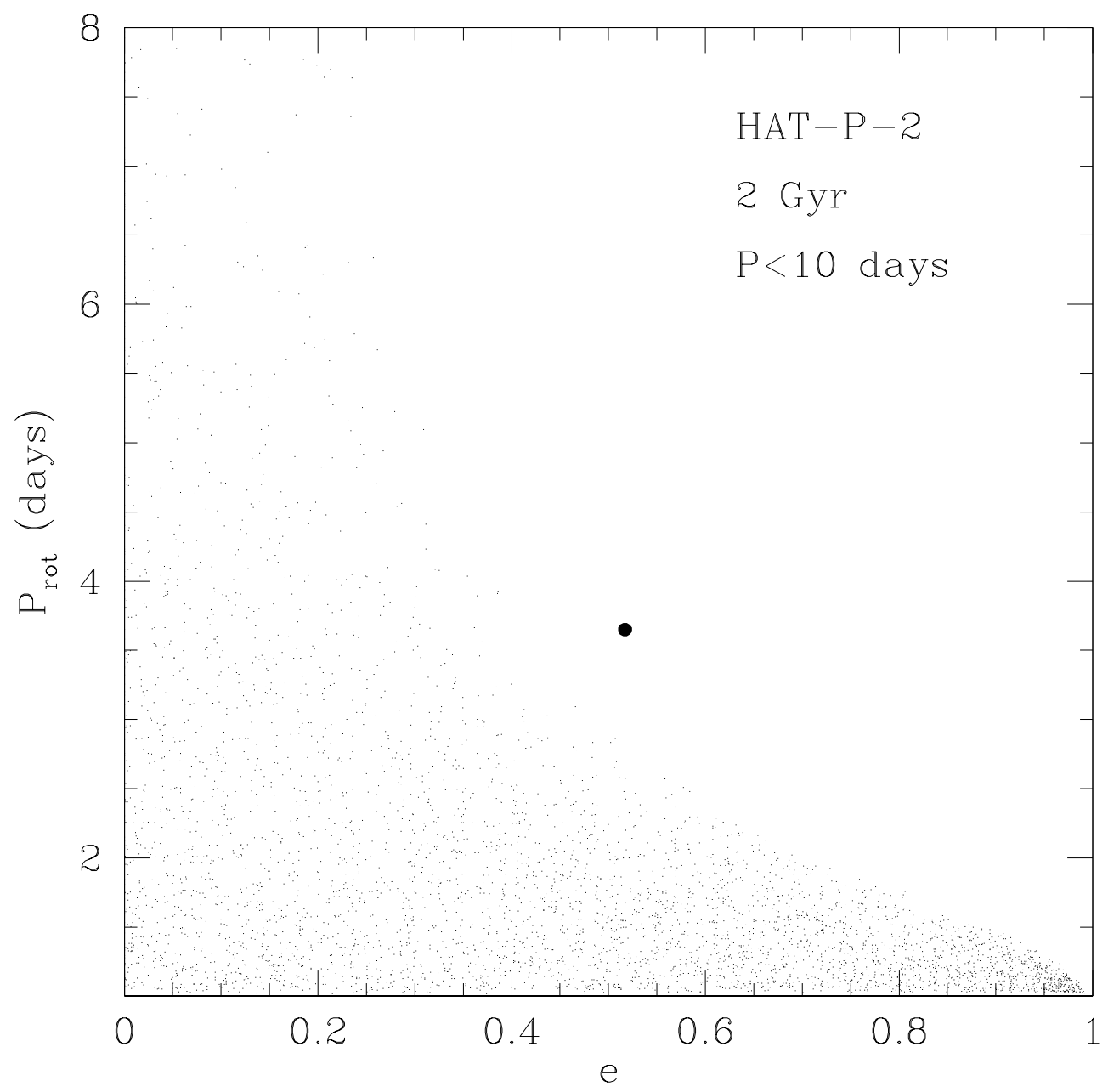




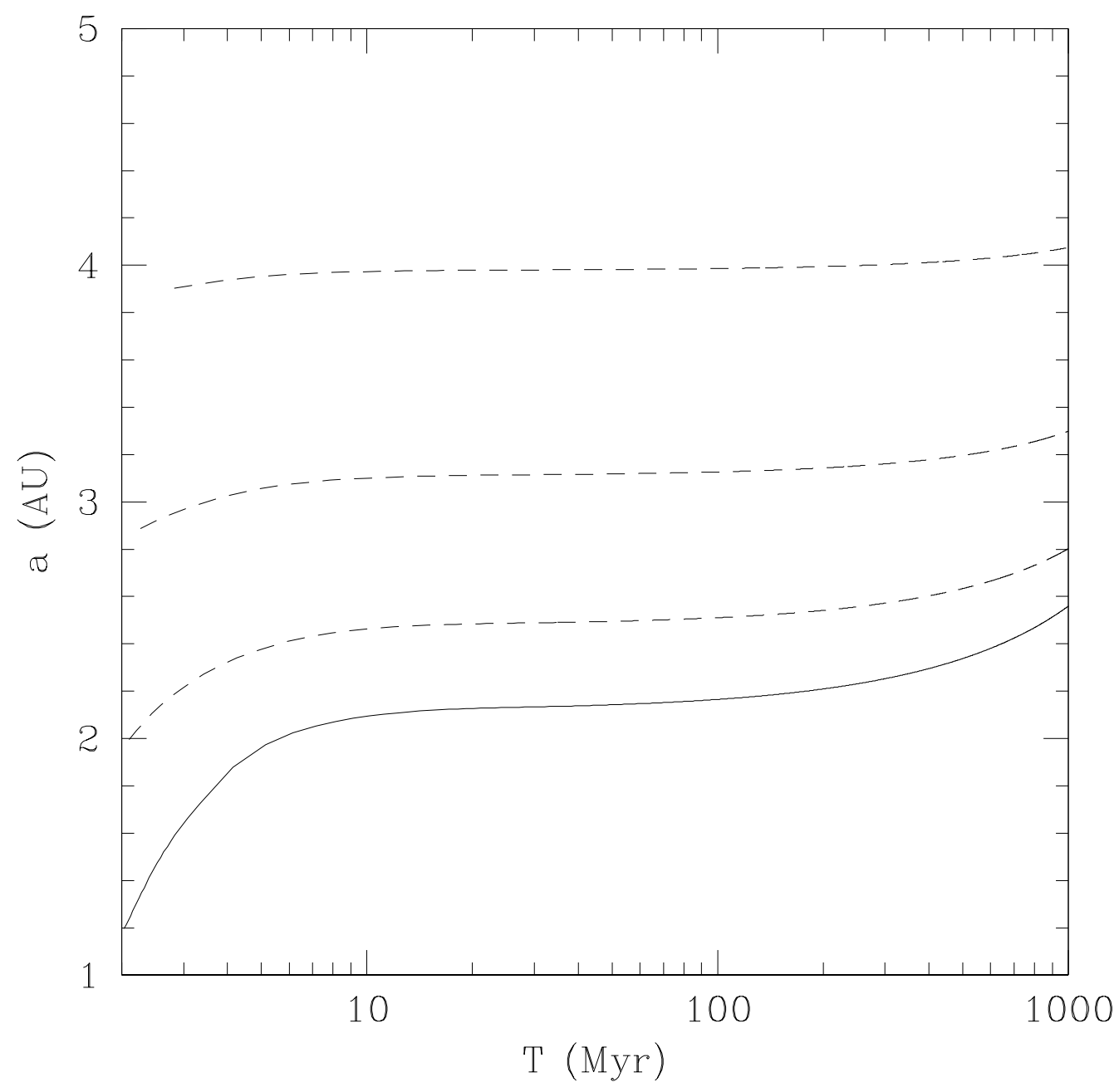



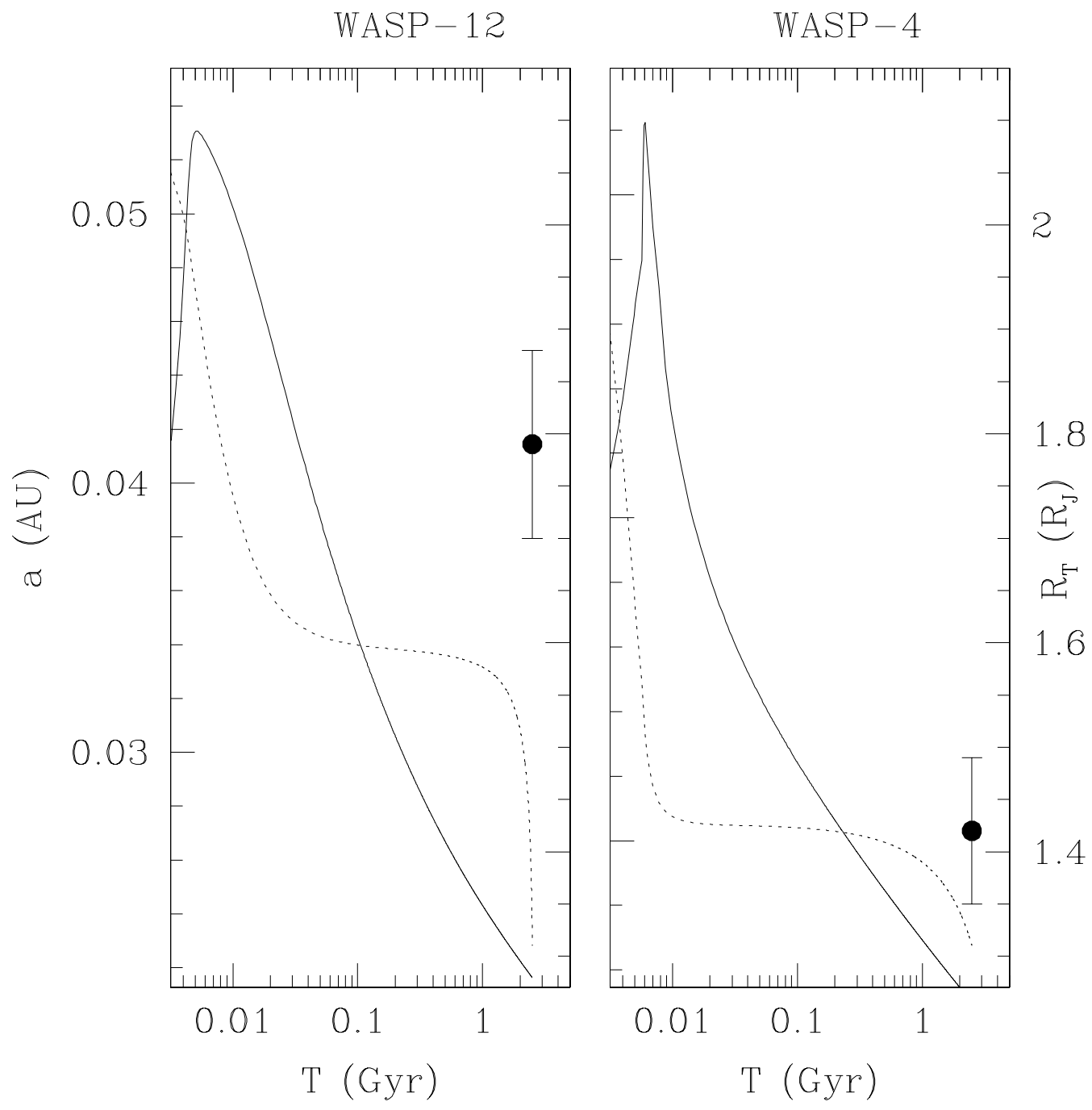


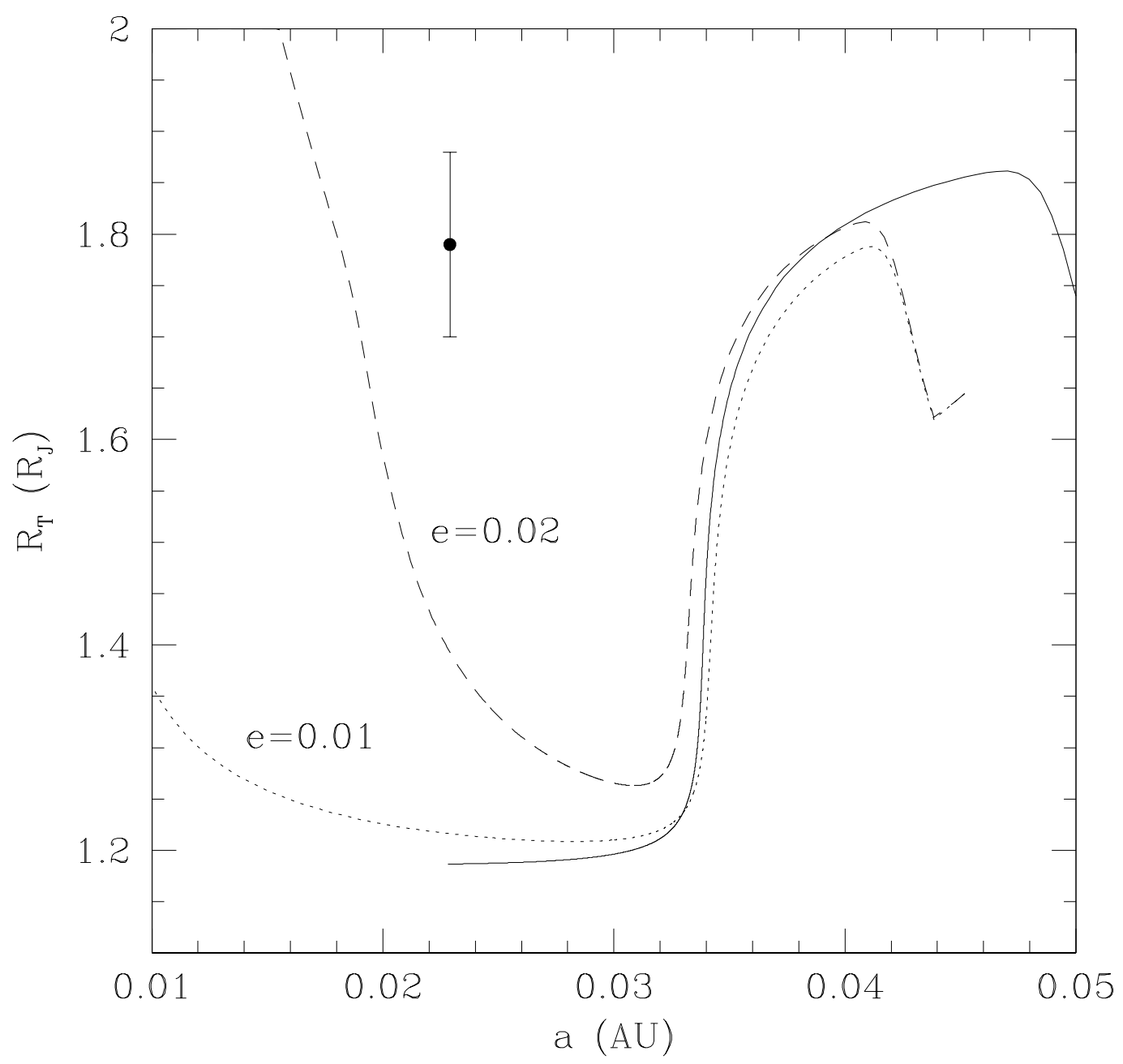




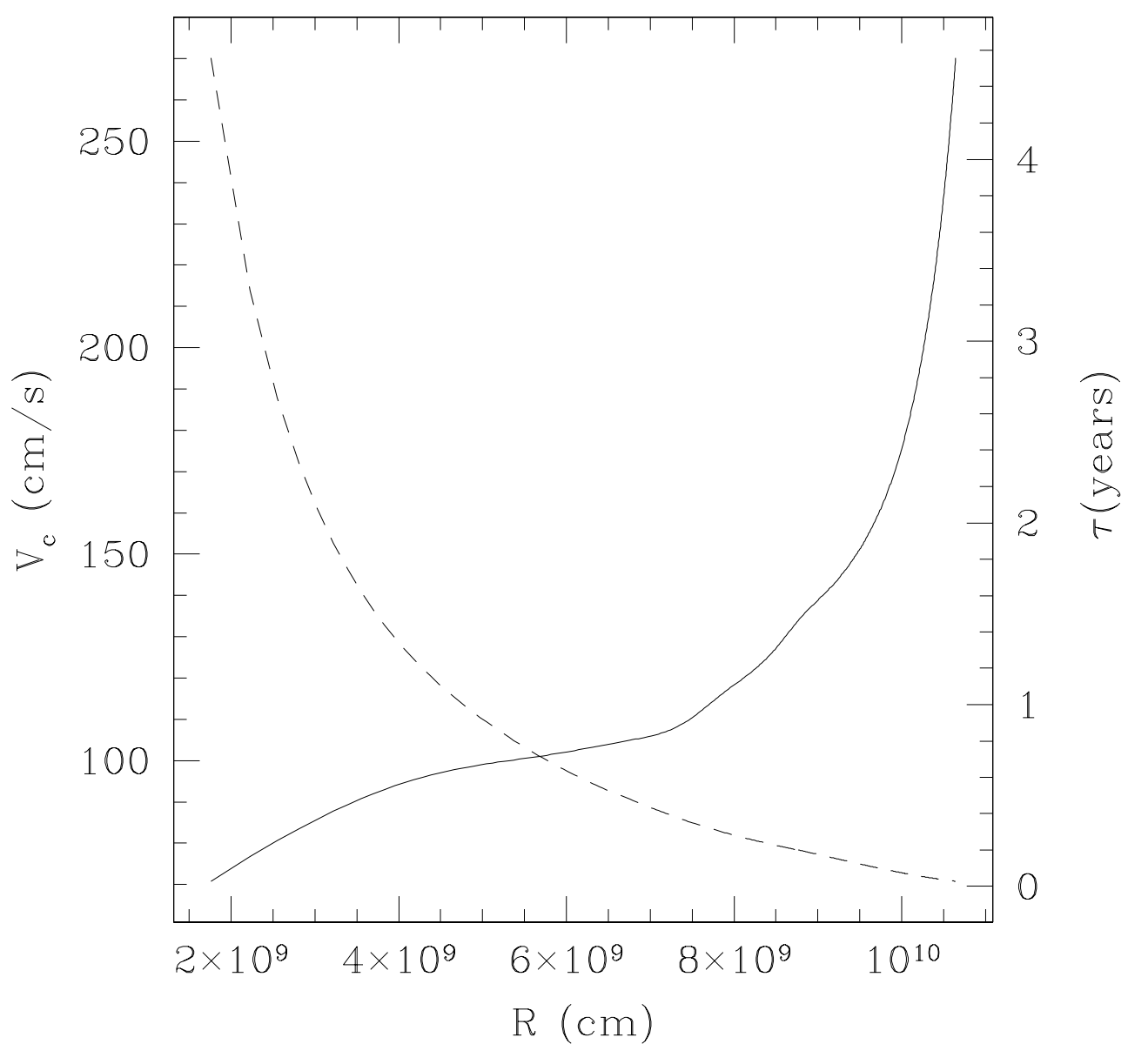




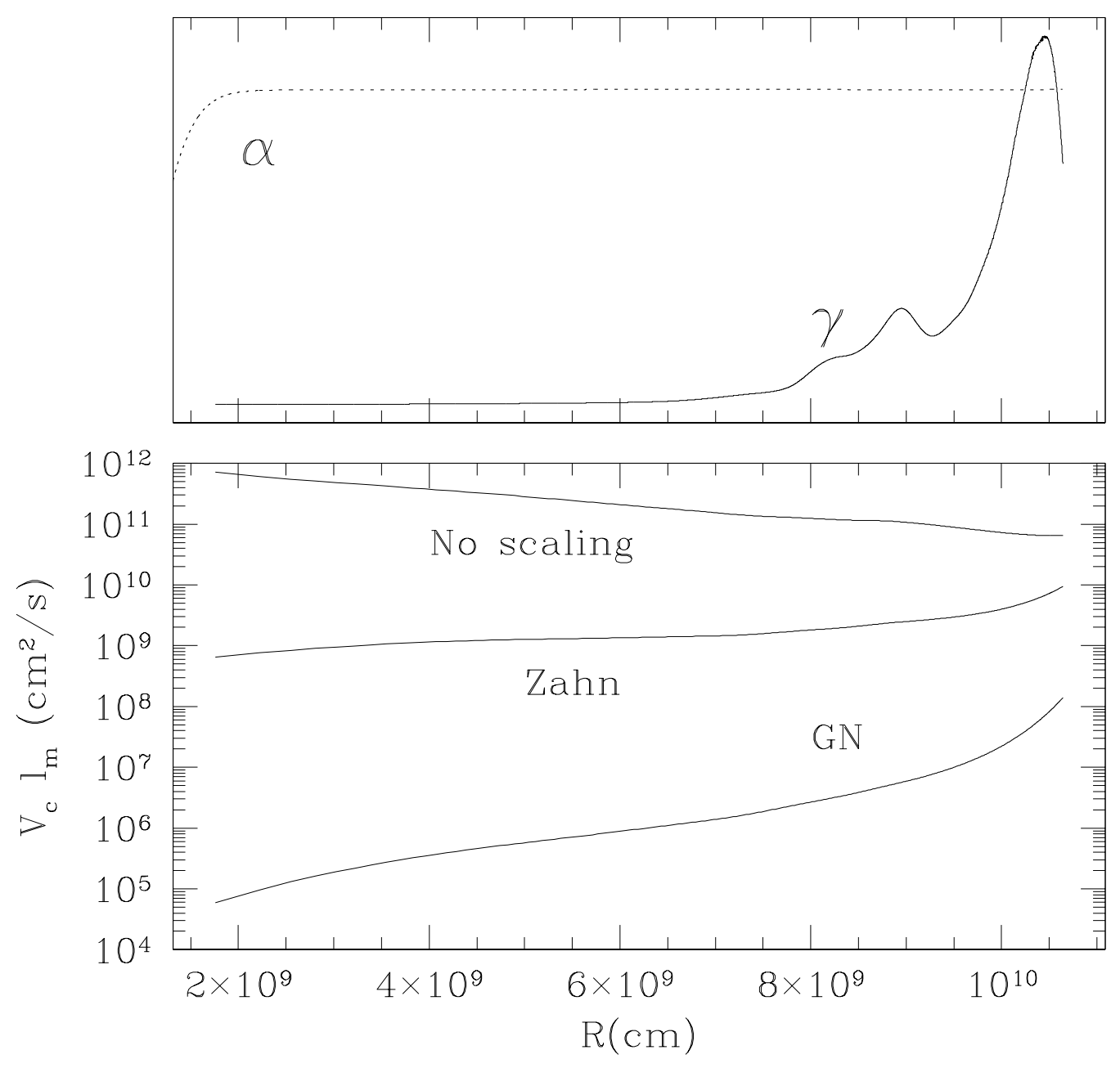




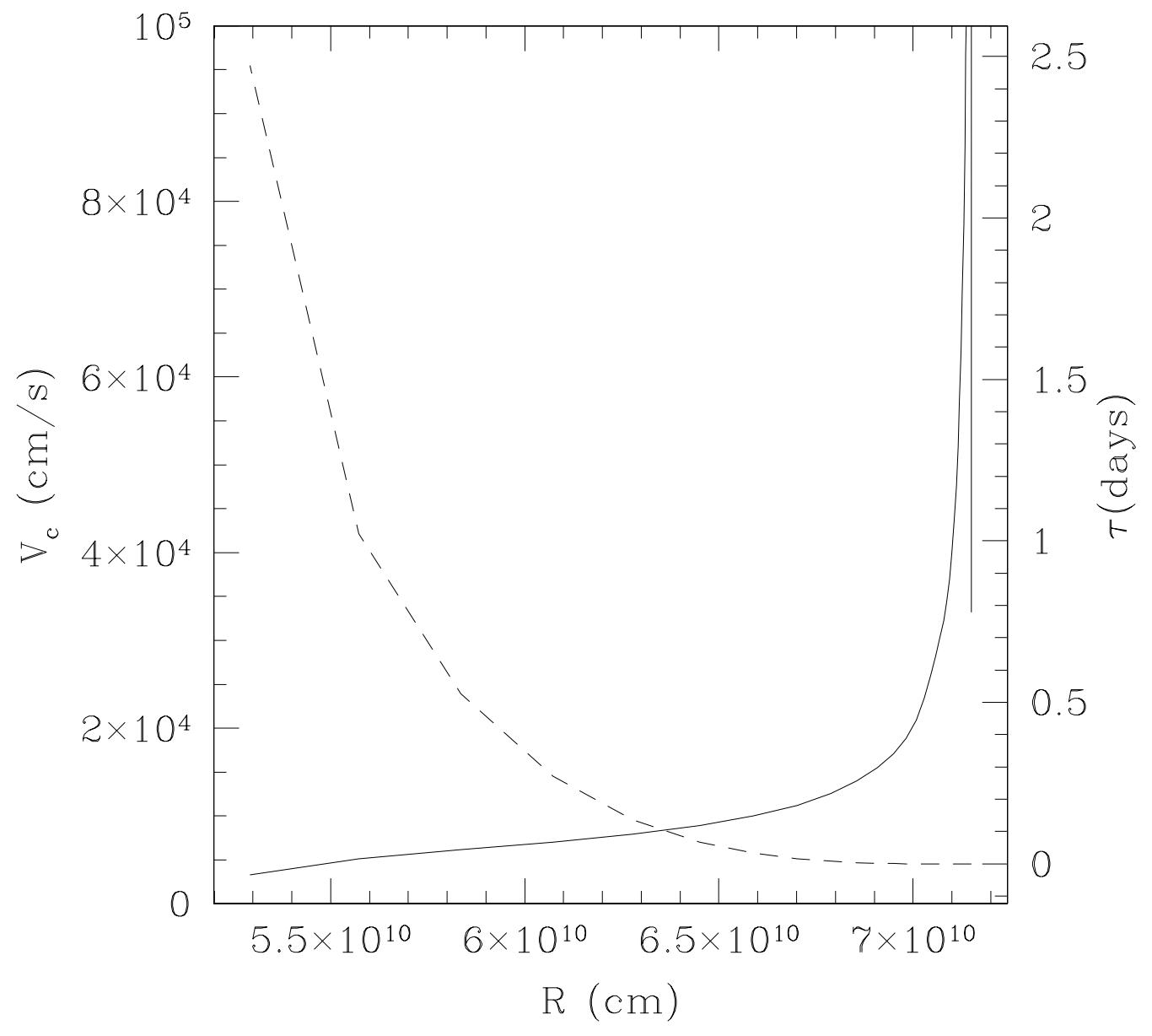

